\title{
Review Article \\ Prediction of Spectral Phonon Mean Free Path and Thermal Conductivity with Applications to Thermoelectrics and Thermal Management: A Review
}

\author{
Tianli Feng and Xiulin Ruan \\ School of Mechanical Engineering and the Birck Nanotechnology Center, Purdue University, West Lafayette, IN 47907-2088, USA \\ Correspondence should be addressed to Xiulin Ruan; ruan@purdue.edu
}

Received 12 November 2013; Accepted 16 January 2014; Published 31 March 2014

Academic Editor: Urszula Narkiewicz

Copyright (C) 2014 T. Feng and X. Ruan. This is an open access article distributed under the Creative Commons Attribution License, which permits unrestricted use, distribution, and reproduction in any medium, provided the original work is properly cited.

We give a review of the theoretical approaches for predicting spectral phonon mean free path and thermal conductivity of solids. The methods can be summarized into two categories: anharmonic lattice dynamics calculation and molecular dynamics simulation. In the anharmonic lattice dynamics calculation, the anharmonic force constants are used first to calculate the phonon scattering rates, and then the Boltzmann transport equations are solved using either standard single mode relaxation time approximation or the Iterative Scheme method for the thermal conductivity. The MD method involves the time domain or frequency domain normal mode analysis. We present the theoretical frameworks of the methods for the prediction of phonon dispersion, spectral phonon relaxation time, and thermal conductivity of pure bulk materials, layer and tube structures, nanowires, defective materials, and superlattices. Several examples of their applications in thermal management and thermoelectric materials are given. The strength and limitations of these methods are compared in several different aspects. For more efficient and accurate predictions, the improvements of those methods are still needed.

\section{Introduction}

In recent years, increasing attention has been focused on seeking novel structures and materials with desired thermal properties, especially thermal conductivity. High thermal conductivity can help remove heat rapidly and reduce device temperatures so as to improve performance of nanoelectronics and optoelectronics, while low thermal conductivity is desired in thermoelectrics for improving the figures of merit $Z T$ [1] of the material: $Z T=S^{2} \sigma T / k$, where $S, \sigma, T$, and $k$ are Seebeck coefficient, electronic conductivity, temperature, and thermal conductivity, respectively. The thermal conductivity $k$ is a summation of the lattice contribution $k_{l}$ and electron contribution $k_{e}$. Since, in most thermoelectric materials, the phonon mean free path is much longer than that of electrons, one major strategy to enhance $Z T$ is to reduce $k_{l}$ without much affecting $k_{e}$. This is made possible by the rapid development of nanofabrication techniques.

Gaining a deeper physical insight into the spectral phonon properties, for example, the spectral phonon relaxation time and mean free path, is necessary to correctly explain experimental results and accurately predict and guide the further designs and applications. Analytical models have been used by Balandin and Wang to estimate frequency-dependent phonon group velocity and various phonon scattering rates including phonon-phonon, phononimpurity, and phonon-boundary scattering processes. They used this approach to observe the strong modification of acoustic phonon group velocity and enhanced phonon scattering rate due to boundary scattering in semiconductor quantum wells, so as to successfully explain their significantly reduced lattice thermal conductivity [2]. This effect of phonon confinement was then extended to nanowires and quantum dot superlattices [3-5]. Analytical models of spectral phonon properties are advantageous in their clear physical insights, but they usually contain empirical fitting parameters, and this limitation has motivated the development of numerical methods based on first principles and molecular dynamics that can predict these spectral properties from their atomic structure, without fitting 
parameters and with greater accuracy. This review will be focused on these predictive simulation methods.

The methods of predicting spectral phonon relaxation times and mean free paths become increasingly important for predicting the thermal properties of numerous novel materials. For instance, superlattice structure is found to be an effective way to suppress the thermal conductivity because of the interface mass mismatch scattering [6-8], but the phenomenon in which the short-period superlattice can have even higher thermal conductivity still needs the deep insight of phonon relaxation time. Doping and alloying are widely used to explore novel high performance materials [9], and the natural materials are rarely pure; thus the study of impurity scattering contributing to phonon relaxation time is important. Layer and tube structured materials, for example, graphene [10-13] and carbon nanotube (CNT) [1417], are proved to have unusual phonon transport features such as high thermal conductivity [18-20], which still need to be further understood. Comprehensive reviews of their thermal transport can be found in [21-23]. Nanowires are most commonly studied and used in both theoretical and experimental research [24-32], and the accurate prediction of thermal conductivity needs the knowledge of spectral phonon scattering by boundaries.

Many methods have been proposed and applied to predict spectral phonon relaxation time in the last half century. At the earliest, Klemens and other researchers obtained the frequency-dependent phonon relaxation time mostly by long-wave approximation (LWA) and Deybe model: Klemens obtained the phonon relaxation times by Umklapp $(U)$ three-phonon scattering [33,34] and defect scattering [35], Herring studied normal $(N)$ three-phonon scattering [36], Holland extended the results to dispersive transverse mode range [37], and Casimir studied boundary scattering [3840]. A more accurate method, the third-order anharmonic lattice dynamics (ALD) calculation which can predict the intrinsic spectral phonon relaxation times without LWA, was presented by Maradudin and the coworkers [41, 42]. ALD methods were then applied to silicon and germanium by $a b$ initio approach first by Debernardi et al. [43] and Deinzer et al. [44]. Beyond the standard ALD calculation, Omini and Sparavigna $[45,46]$ proposed an Iterative Scheme which gives exact solutions to the linearized Boltzmann transport equation (BTE). The Iterative Scheme has been successfully applied to many structures in the recent ten years by Broido, Lindsay, Ward, and so forth [47-65]. Other than the lattice dynamics calculation, a time domain normal mode analysis (NMA) method based on molecular dynamics (MD) simulation was proposed by Ladd et al. [66] and extended by McGaughey and Kaviany [67]. Another version of normal mode analysis is implemented in frequency domain, so called spectral energy density (SED) analysis. The normal mode analysis was early implemented by Wang et al. [68-75] to obtain the relaxation times of a few phonon modes and then extended by de Koker [76] and Thomas et al. [77, 78] to calculate lattice thermal conductivity.

In this work, we present a review of the methods of predicting spectral phonon properties, discuss the applications to each method, and compare them in different aspects.
Section 2 gives an overview of thermal conductivity and the frequency-dependent relaxation time predicted from early long-wave approximation (LWA) and Debye model. Section 3 presents the ALD calculation which is divided into three subsections: Section 3.1 covers the standard single mode relaxation time approximation (SMRTA), Section 3.2 gives the Iterative Scheme, and Section 3.3 reviews examples of the applications to pure bulk, layer and tube structures, nanowires, defective materials, and superlattice. In Section 4, we introduce the time domain NMA and frequency NMA methods based on MD simulations and their applications. The summary is presented in Section 5. The appendix provides some derivations of ALD methods.

\section{Theory Overview}

Spectral phonon mean free path (MFP), determined by phonon scattering rate, dominates the behavior of thermal properties, especially the thermal conductivity $k$. Based on BTE under the relaxation time approximation (RTA), thermal conductivity is determined by the spectral phonon relaxation time $\tau_{\lambda}$, phonon group velocity $\mathbf{v}_{\lambda}=\nabla_{\mathbf{k}} \omega$, and phonon specific heat $c_{\lambda}[34]$ :

$$
k_{z}=\frac{1}{V} \sum_{\lambda}\left(\mathbf{v}_{\lambda} \cdot \widehat{z}\right)^{2} c_{\lambda} \tau_{\lambda},
$$

where $\widehat{z}$ denotes the transport direction, $\lambda$ is the shorthand of phonon mode $(\mathbf{k}, \nu)$ with $\mathbf{k}$ representing the phonon wave vector and $v$ labeling phonon dispersion branch, $V$ is the volume of the domain, and the summation is done over the resolvable phonon modes in the domain. The specific heat per mode is $c_{\lambda}=\hbar \omega_{\lambda} \partial n_{\lambda}^{0} / \partial T=k_{B} x^{2} e^{x} /\left(e^{x}-1\right)^{2}$, where $n_{\lambda}^{0}$ is phonon occupation number of the Bose-Einstein distribution $n_{\lambda}^{0}=\left(e^{x}-1\right)^{-1}$ and $x$ is the shorthand of $\hbar \omega / k_{B} T$. Equation (1) can also be expressed in terms of phonon mean free path $\vec{\Lambda}_{\lambda}=\mathbf{v}_{\lambda} \tau_{\lambda}$. The continuous form of (1) is, with the help of $\sum_{\mathbf{k}}=\left(V /(2 \pi)^{3}\right) \int d \mathbf{k}$,

$$
k_{z}=\frac{1}{(2 \pi)^{3}} \sum_{\nu} \int\left(\mathbf{v}_{\lambda} \cdot \widehat{z}\right)^{2} c_{\lambda} \tau_{\lambda} d \mathbf{k} .
$$

If isotropic heat transport is assumed, the integration of $\left|v_{\lambda, z}\right|^{2}$ in (2) gives $v_{\lambda}^{2} / 3$, and $\int d \mathbf{k}$ gives $\int 4 \pi k^{2} d k$, we get the commonly used formula

$$
k_{z}=\frac{4 \pi}{3} \frac{1}{(2 \pi)^{3}} \sum_{\nu} \int c_{\lambda} v_{\lambda} \Lambda_{\lambda} k^{2} d k .
$$

The early theoretical predictions of phonon relaxation times for different scattering processes are briefly summarized in Table $1[37,79] . T$ is temperature; subscripts $N, U$, $T$, and $L$ indicate the Umklapp scattering, normal scattering, transverse wave, and longitudinal wave, respectively; $A, B$ 's, and $C$ 's are constants; $\theta$ is Debye temperature; $\alpha$ is numerical constant in [34]; Low $T$ means $T \ll \theta$, and high $T$ means $T \gg$ $\theta$. $\omega_{1}$ is the transverse mode frequency at which the group velocity starts to decrease, and $\omega_{2}$ is the maximum transverse 
TABLE 1: Analytical models of inverse relaxation time for different scattering processes.

\begin{tabular}{|c|c|}
\hline Scattering process & Inverse relaxation time \\
\hline \multicolumn{2}{|l|}{ Intrinsic Three-Phonon } \\
\hline \multicolumn{2}{|l|}{$N$ process } \\
\hline Herring $^{\mathrm{a}}$ & $\begin{array}{l}\tau_{L, N}^{-1}=B_{L} \omega^{2} T^{3}, \text { low } T \\
\tau_{T, N}^{-1}=B_{T} \omega T^{4}, \text { low } T \\
\tau_{L, N}^{-1}=B_{L}^{\prime} \omega^{2} T, \text { high } T \\
\tau_{T, N}^{-1}=B_{T}^{\prime} \omega T, \text { high } T\end{array}$ \\
\hline Callaway $^{\mathrm{b}}$ & $\tau_{N}^{-1}=B_{N} \omega^{2} T^{3}$ \\
\hline \multicolumn{2}{|l|}{$U$ process } \\
\hline Klemens $^{c}$ & $\tau_{U}^{-1}=B_{U} \omega^{2} T^{3} \exp \left(-\frac{\theta}{\alpha T}\right)$, low $T$ \\
\hline Klemens $^{\mathrm{d}}$ & $\begin{aligned} \tau_{U}^{-1} & =B_{U} \omega T^{3} \exp \left(-\frac{\theta}{\alpha T}\right), \text { low } T \\
\tau_{U}^{-1} & =B^{\prime} \omega^{2} T, \operatorname{high} T\end{aligned}$ \\
\hline Callaway $^{\mathrm{b}}$ & $\begin{array}{r}\tau_{U}^{-1}=B_{U} \omega^{2} T^{3} \\
B_{T U} \omega^{2}\end{array}$ \\
\hline Holland ${ }^{\mathrm{e}}$ & $\begin{array}{c}\tau_{T, U}^{-1}=\frac{\sigma^{T U}}{\sinh (x)}, \omega_{1} \leq \omega \leq \omega_{2} \\
0, \quad \omega<\omega_{1}\end{array}$ \\
\hline Asen-Palmer et al. ${ }^{\mathrm{f}}$ & $\begin{aligned} \tau_{T, U}^{-1} & =B_{T U}^{\prime} \omega^{2} T \exp \left(\frac{C_{T}}{T}\right) \\
\tau_{L, U}^{-1} & =B_{L U}^{\prime} \omega^{2} T \exp \left(\frac{C_{L}}{T}\right)\end{aligned}$ \\
\hline Boundary ${ }^{\mathrm{g}}$ & $\begin{array}{c}\tau_{b}^{-1}=\frac{v_{\lambda}}{l F} \quad l: \text { diameter, } l=2 \sqrt{l_{1} l_{2} / \pi} \\
F: \text { surface roughmess }\end{array}$ \\
\hline Impurity $^{\mathrm{h}}$ & $\tau_{\mathrm{im}}^{-1}=\frac{\pi}{2} g \omega^{2} D(\omega) \approx \frac{V_{0} g}{4 \pi\left\langle v^{3}\right\rangle} \omega^{4} \sim A \omega^{4}$ \\
\hline
\end{tabular}

References: ${ }^{\mathrm{a}}[36],{ }^{\mathrm{b}}[80],{ }^{\mathrm{c}}[33],{ }^{\mathrm{d}}[34],{ }^{\mathrm{e}}[37],{ }^{\mathrm{f}}[81],{ }^{\mathrm{g}}[38-40]$, and ${ }^{\mathrm{h}}[35]$.

frequency. The intrinsic three-phonon scattering rates are derived mostly in LWA or linear dispersion approximation.

Boundary scattering $\tau_{b}^{-1}$ exists anywhere, since every sample has a finite size. $F$ captures the boundary scattering characteristic of the sample with $F=1$ representing completely diffusive and $F \rightarrow \infty$ meaning specular. $l=$ $2 \sqrt{S_{c} / \pi}$ is a measure of the size perpendicular to the transport direction, with $S_{c}$ being the area of cross section. $v_{\lambda}$ is often replaced by the average phonon speed of the three acoustic branches $v_{\text {ave }}$ for simplicity [37]:

$$
v_{\mathrm{ave}}=\left[\frac{1}{3} \sum_{\nu}^{3} \frac{1}{v_{v}}\right]^{-1}=\left[\frac{1}{3}\left(\frac{2}{v_{T}}+\frac{1}{v_{L}}\right)\right]^{-1} .
$$

The last equation in Table 1 takes into account the impurity scattering rate, where

$$
g=\sum_{i} f_{i}\left(1-\frac{m_{i}}{\bar{m}}\right)^{2}
$$

is a measure of mass disorder, $D(\omega)$ is phonon density of states normalized to unity, $f_{i}$ is the concentration of the impurity species $i$, and $m_{i}$ and $\bar{m}$ are the mass of $i$ and average mass for the given composition, respectively. The exact expression for $\left\langle v^{3}\right\rangle$ is found in [82], while, in long wave approximation, $\left\langle v^{3}\right\rangle$ approximates the cube of acoustic phonon speed of the material. This equation was derived by Klemens for isotope scattering with only mass disorder. For crystal defects other than isotope doping, such as vacancy, interstitial, and antisite defects, the impurity scattering comes from not only the mass disorder but also the interatomic force change and link break. Klemens took into account such effect by adding a modification to $g$ :

$$
\tilde{g}=g+2 \sum_{i} f_{i}\left(\frac{\Delta \phi_{i}}{\phi}-\frac{6.4 \gamma \Delta r_{i}}{r}\right)^{2},
$$

where $\phi_{i} / \phi$, and $\Delta r_{i} / r$ describe the average relative variations of the local force constants and atomic displacements $[35,83-$ 85], respectively. Some consider the dislocations by adding a scattering term $\tau_{D}^{-1} \sim \omega$ to the total phonon scattering rate [84], predicted from single dislocation assumption by $[34,35$, 86, 87]. Although $\tau_{\text {im }}^{-1}=A \omega^{4}$ is derived for low frequency phonons, many works use it to predict thermal conductivity or explain data from experiments for alloys and crystals with impurities [24-26, 37, 81, 84, 85, 88-91]. In Section 3.3.4, we will give more precise expressions for isotope scattering.

For the system that contains several scattering mechanisms, the Matthiessen rule is often used to evaluate the total scattering rate,

$$
\tau^{-1}=\sum_{i} \tau_{i}^{-1}
$$

In most cases the Matthiessen rule gives reasonable results, although it is found to be not accurate in some cases recently $[58,92,93]$.

These frequency dependent relaxation time expressions in Table 1 have been used in many works for thermal conductivity prediction and analysis, and the choice of those expressions looks quite arbitrary. For instance, in the choice of intrinsic phonon relaxation time in the thermal conductivity analysis of silicon, Glassbrenner and Slack [94] used $\tau^{-1} \sim$ $\omega^{2} T$, while Asen-Palmer et al. [81] and Mingo et al. [24, 25] used $\tau^{-1} \sim \omega^{2} T \exp (C / T)$ for all phonon modes; Martin et al. [26] used $\tau_{L}^{-1} \sim \omega^{2} T^{3}$ for longitudinal mode, while Holland added $\tau_{T}^{-1} \sim \omega^{2} / \sinh (x)$ to dispersive transverse range. The thermal conductivity results predicted by these expressions can be reasonable due to the adjustable fitting parameters. Therefore, it becomes important to accurately predict spectral phonon relaxation time without any fitting parameter, which allows us to understand thermal transport and examine (a) the validity of low-frequency approximation or the Debye model, (b) the importance of optical branch to thermal transport, (c) the contributions of phonons with different mean free path or different wavelength to thermal conductivity, (d) the relative importance of different scattering mechanisms in a given material, and so forth.

\section{Anharmonic Lattice Dynamics Methods}

In perturbation theory, the steady-state phonon BTE [34, 79,95 ] describes the balance of phonon population between diffusive drift and scattering as

$$
\mathbf{v}_{\lambda} \cdot \nabla n_{\lambda}=\left.\frac{\partial n_{\lambda}}{\partial t}\right|_{s},
$$


where $n_{\lambda}=n_{\lambda}^{0}+n_{\lambda}^{\prime}$ is the total phonon occupation number with $n_{\lambda}^{\prime}$ representing the deviation from the equilibrium phonon distribution $n_{\lambda}^{0}$. With $\nabla n_{\lambda}=\left(\partial n_{\lambda} / \partial T\right) \nabla T$ and assuming that $n_{\lambda}^{\prime}$ is independent of temperature: $\left(\partial n_{\lambda} / \partial T\right) \simeq$ $\left(\partial n_{\lambda}^{0} / \partial T\right)$, we have

$$
\mathbf{v}_{\lambda} \cdot \nabla T \frac{\partial n_{\lambda}^{0}}{\partial T}=\left.\frac{\partial n_{\lambda}^{\prime}}{\partial t}\right|_{s}
$$

The RTA assumes that deviation of single phonon mode population decays exponentially with time:

$$
n_{\lambda}^{\prime} \sim \exp \left(-\frac{t}{\tau_{\lambda}}\right),
$$

where $\tau_{\lambda}$ is the relaxation time. Therefore, the collision term in BTE (9) becomes

$$
\left.\frac{\partial n_{\lambda}^{\prime}}{\partial t}\right|_{s} \simeq-\frac{n_{\lambda}^{\prime}}{\tau_{\lambda}}
$$

Generally, the value of $\tau_{\lambda}$ is considered as the average time between collisions of the phonon mode $\lambda$ with other modes, whereby $\tau_{\lambda}=1 / \Gamma_{\lambda}$, where $\Gamma_{\lambda}$ denotes the scatting rate.

Considering only three-phonon scattering, (9) becomes [95]

$$
\begin{aligned}
& \mathbf{v}_{\lambda} \nabla T \frac{\partial n_{\lambda}^{0}}{\partial T} \\
&=-\sum_{\lambda^{\prime} \lambda^{\prime \prime}}\left\{\left[n_{\lambda} n_{\lambda^{\prime}}\left(1+n_{\lambda^{\prime \prime}}\right)-\left(1+n_{\lambda}\right)\left(1+n_{\lambda^{\prime}}\right) n_{\lambda^{\prime \prime}}\right] \mathfrak{I}_{+}\right. \\
&\left.\quad+\frac{1}{2}\left[n_{\lambda}\left(1+n_{\lambda^{\prime}}\right)\left(1+n_{\lambda^{\prime \prime}}\right)-\left(1+n_{\lambda}\right) n_{\lambda^{\prime}} n_{\lambda^{\prime \prime}}\right] \mathfrak{Q}_{-}\right\},
\end{aligned}
$$

where the summation is done over all the phonon modes $\lambda^{\prime}$ and $\lambda^{\prime \prime}$ that obey the energy conservation $\omega_{\lambda} \pm \omega_{\lambda^{\prime}}=\omega_{\lambda^{\prime \prime}}$ and quasimomentum conservation $\mathbf{k} \pm \mathbf{k}^{\prime}=\mathbf{k}^{\prime \prime}+\mathbf{G}$ with $\mathbf{G}=$ $\mathbf{0}$ for $N$ processes and $\mathbf{G} \neq \mathbf{0}$ for $U$ processes, where $\mathbf{G}$ is a reciprocal-lattice vector. $\mathfrak{Q}_{+}$is the probability of $\lambda \pm \lambda^{\prime} \rightarrow \lambda^{\prime \prime}$ scattering occurrence, determined via Fermi's golden rule

$$
\begin{gathered}
\mathfrak{Q}_{ \pm}=\frac{\hbar \pi}{4 N_{0}}\left|V_{ \pm}^{(3)}\right|^{2} \frac{\delta\left(\omega_{\lambda} \pm \omega_{\lambda^{\prime}}-\omega_{\lambda^{\prime \prime}}\right)}{\omega_{\lambda} \omega_{\lambda^{\prime}} \omega_{\lambda^{\prime \prime}}} \\
V_{ \pm}^{(3)}=\sum_{b l^{\prime} b^{\prime} l^{\prime \prime} b^{\prime \prime}} \sum_{\alpha \beta \gamma} \Phi_{0 b, l^{\prime} b^{\prime}, l^{\prime \prime} b^{\prime \prime}}^{\alpha \beta \gamma} \frac{e_{\alpha b}^{\lambda} e_{\beta b^{\prime}}^{ \pm \lambda^{\prime}} e_{\gamma b^{\prime \prime}}^{-\lambda^{\prime \prime}}}{\sqrt{\bar{m}_{b} \bar{m}_{b^{\prime}} \bar{m}_{b^{\prime \prime}}}} e^{ \pm i \mathbf{k}^{\prime} \cdot \mathbf{r}_{l^{\prime}}} e^{-i \mathbf{k}^{\prime \prime} \cdot \mathbf{r}_{l^{\prime \prime}}},
\end{gathered}
$$

where $b$ 's and $l$ 's are the indexes of basis atoms and unit cells, respectively, $\alpha, \beta$, and $\gamma$ represent coordinate directions, $m_{b}$ is the mass of basis atom $b$, considering that some doping material $\bar{m}_{b}$ is the average mass in the $b$ th basis sites, $e_{b, \alpha}^{\lambda}$ is the $\alpha$ component of the $b$ th part of the mode $\lambda \stackrel{b, \alpha}{=}$ $(\mathbf{k}, \nu)$ 's eigenvector, and $\Phi$ is the third-order interatomic force constant (IFC). The factor " $1 / 2$ " in (12) accounts for the double counting in the summation of $\lambda^{\prime}$ and $\lambda^{\prime \prime}$ for the "-" process. In (14), the factor $e^{i \mathbf{k} \cdot \mathbf{r}_{l}}$ is often omitted, since it is a constant in the summation and thus contributes nothing to $\left|V_{ \pm}^{(3)}\right|^{2}$.

3.1. Standard Single Mode Relaxation Time Approximation. The Standard SMRTA assumes that the system is in its complete thermal equilibrium, except that one phonon mode $\lambda$ has its occupation number $n_{\lambda}=n_{\lambda}^{0}+n_{\lambda}^{\prime}$ differing a small amount from its equilibrium value $n_{\lambda}^{0}$. Therefore, on the right hand side of (12), replacing $n_{\lambda}$ by $n_{\lambda}^{0}+n_{\lambda}^{\prime}$, whilst $n_{\lambda^{\prime}}$ and $n_{\lambda^{\prime \prime}}$ by $n_{\lambda^{\prime}}^{0}$ and $n_{\lambda^{\prime \prime}}^{0}$, respectively, one can obtain the phonon relaxation time $\tau_{\lambda}^{0}$ of mode $\lambda$ (for the derivation, see Appendix A.1):

$$
\frac{1}{\tau_{\lambda}^{0}}=\sum_{\lambda^{\prime} \lambda^{\prime \prime}}^{+} \Gamma_{\lambda \lambda^{\prime} \lambda^{\prime \prime}}^{+}+\frac{1}{2} \sum_{\lambda^{\prime} \lambda^{\prime \prime}}^{-} \Gamma_{\lambda \lambda^{\prime} \lambda^{\prime \prime}}^{-}+\sum_{\lambda^{\prime}} \Gamma_{\lambda \lambda^{\prime}}^{\mathrm{ext}}
$$

where the first two terms on the right hand side are intrinsic three-phonon scattering rates $\left(\omega_{\lambda} \pm \omega_{\lambda^{\prime}}=\omega_{\lambda^{\prime \prime}}\right)$ :

$$
\begin{aligned}
\Gamma_{\lambda \lambda^{\prime} \lambda^{\prime \prime}}^{ \pm}= & \frac{\hbar \pi}{4 N_{0}}\left\{\begin{array}{c}
n_{\lambda^{\prime}}^{0}-n_{\lambda^{\prime \prime}}^{0} \\
n_{\lambda^{\prime}}^{0}+n_{\lambda^{\prime \prime}}^{0}+1
\end{array}\right\} \\
& \times\left|V_{ \pm}^{(3)}\right|^{2} \frac{\delta\left(\omega_{\lambda} \pm \omega_{\lambda^{\prime}}-\omega_{\lambda^{\prime \prime}}\right)}{\omega_{\lambda} \omega_{\lambda^{\prime}} \omega_{\lambda^{\prime \prime}}} .
\end{aligned}
$$

The last term $\Gamma_{\lambda \lambda^{\prime}}^{\text {ext }}$ represents the extrinsic scattering such as boundary scattering and impurity scattering.

3.2. Iterative Scheme: Exact Solution to Linearized BTE. Different from the Standard SMRTA, the other method to solve the phonon BTE allows all the modes to be in their thermal nonequilibrium states at the same time. By replacing the occupation numbers $n_{\lambda}, n_{\lambda^{\prime}}$, and $n_{\lambda^{\prime \prime}}$ by $n_{\lambda}^{0}+n_{\lambda}^{\prime}, n_{\lambda^{\prime}}^{0}+n_{\lambda^{\prime}}^{\prime}$, and $n_{\lambda^{\prime \prime}}^{0}+n_{\lambda^{\prime \prime}}^{\prime}$, respectively, on the right hand side of (12), the relaxation time $\tau_{\lambda}$ of mode $\lambda$ is obtained (for the derivation see Appendix A.2)

$$
\begin{gathered}
\tau_{\lambda}=\tau_{\lambda}^{0}\left(1+\Delta_{\lambda}\right), \\
\Delta_{\lambda}=\sum_{\lambda^{\prime} \lambda^{\prime \prime}}^{(+)} \Gamma_{\lambda \lambda^{\prime} \lambda^{\prime \prime}}^{+}\left(\xi_{\lambda \lambda^{\prime \prime}} \tau_{\lambda^{\prime \prime}}-\xi_{\lambda \lambda^{\prime}} \tau_{\lambda^{\prime}}\right) \\
+\sum_{\lambda^{\prime} \lambda^{\prime \prime}}^{(-)} \frac{1}{2} \Gamma_{\lambda \lambda^{\prime} \lambda^{\prime \prime}}^{-}\left(\xi_{\lambda \lambda^{\prime \prime}} \tau_{\lambda^{\prime \prime}}+\xi_{\lambda \lambda^{\prime}} \tau_{\lambda^{\prime}}\right) \\
+\sum_{\lambda^{\prime}} \Gamma_{\lambda \lambda^{\prime}}^{\mathrm{ext}} \xi_{\lambda \lambda^{\prime}} \tau_{\lambda^{\prime}},
\end{gathered}
$$

where $\xi_{\lambda \lambda^{\prime}}=v_{\lambda l, z} \omega_{\lambda^{\prime}} / v_{\lambda, z} \omega_{\lambda}$, and $v_{z}$ is phonon group velocity component along the transport direction.

Equation (17) is solved iteratively because both the left and the right hand sides contain the unknown variable $\tau_{\lambda}$, and thus the method is called Iterative Scheme. This scheme is also based on RTA; thus (10) and (11) are still valid (one can reach this by substituting (A.3), (A.4), (A.11), (A.12), (A.13), and (A.14) into (9)). The last summation in (18) is done over $\lambda^{\prime}$ with $\lambda^{\prime} \neq \lambda$. 
3.3. Discussions and Applications. ALD methods can be divided into classical method and ab initio method, differing in how to calculate the harmonic and anharmonic IFCs, which are the only inputs to these methods. The classical approach relies on empirical interatomic potential whose $n$th order derivatives are taken as the $n$th order IFCs:

$$
\Phi_{l_{1} b_{1}, \ldots, l_{n} b_{n}}^{\alpha_{1} \ldots \alpha_{n}}=\frac{\partial^{n} \Phi}{\partial u_{1}\left(l_{1} b_{1}\right), \ldots, \partial u_{n}\left(l_{n} b_{n}\right)} .
$$

In contrast, the $a b$ initio approach is a first principle calculation in the framework of density functional perturbation theory (DFPT) $[43,96,97]$ using norm-conserving pseudopotentials in the local density approximation (LDA) without introducing any adjustable parameters. The formulism of the IFCs using first principle method can be found in [44] and realized by, for example, the QUANTUM ESPRESSO package [98]. Compared to the classical method, this method can deal with new materials whose empirical interatomic potentials are unknown. Further, this method can be more accurate since the empirical interatomic potentials cannot always represent the exact nature of interatomic force.

In (16), the delta function $\delta\left(\omega \pm \omega^{\prime}-\omega^{\prime \prime}\right)$ is typically approximated by $\delta(x)=\lim _{\varepsilon \rightarrow 0+}(1 / \pi)\left(\varepsilon /\left(x^{2}+\varepsilon^{2}\right)\right)$. To accurately evaluate (16), the choice of $\varepsilon$ value is critical: it must be small but larger than the smallest increment in discrete $x$, which results from the use of finite grid of $k$ points in Brillouin zone. The general practice is as follows: pick the densest grid possible and start with a sufficiently small guess, and increase it gradually until the final results reach convergence.

To calculate the relaxation time, one can use Standard SMRTA scheme $[43,44,99-111]$ or Iterative Scheme [47$65,112]$, and, in each of them, one can choose empirical interatomic potential approach $[47-49,52-56,58-60,99-$ 102] or ab initio-derived IFC IFC [43, 44, 50, 51, 57, 61-65, 103-112]. The methods can be used on pure bulk, nanowires, doped bulk, doped nanowires, alloys, and so forth.

One way to predict thermal conductivity $k$ without working out all the phonon modes relaxation times is the Monte Carlo integration technique [101, 113]. The protocol of this technique is as follows: (1) randomly sample some phonon modes $\lambda$, (2) for each of these modes, randomly choose two other modes $\lambda^{\prime}$ and $\lambda^{\prime \prime}$ that interact with $\lambda$ to calculate the relaxation time, and (3) select as many points as necessary to ensure that the statistical error is small enough in both cases. Monte Carlo technique only works for the Standard SMRTA scheme, since the Iterative Scheme requires the relaxation times of all the phonon modes to do iteration. Monte Carlo technique reduces the computational cost but lowers the accuracy.

In addition to intrinsic phonon scattering $\Gamma^{ \pm}$, extrinsic scattering $1 / \tau_{\lambda}^{\text {ext }}$ plays an important role in nanostructures,

$$
\frac{1}{\tau_{\lambda}^{\mathrm{ext}}}=\sum_{\lambda^{\prime}} \Gamma_{\lambda \lambda^{\prime}}^{\mathrm{ext}}
$$

such as boundary scattering $1 / \tau_{\lambda}^{\text {bs }}$ and impurity scattering $1 / \tau_{\lambda}^{\mathrm{imp}}$.

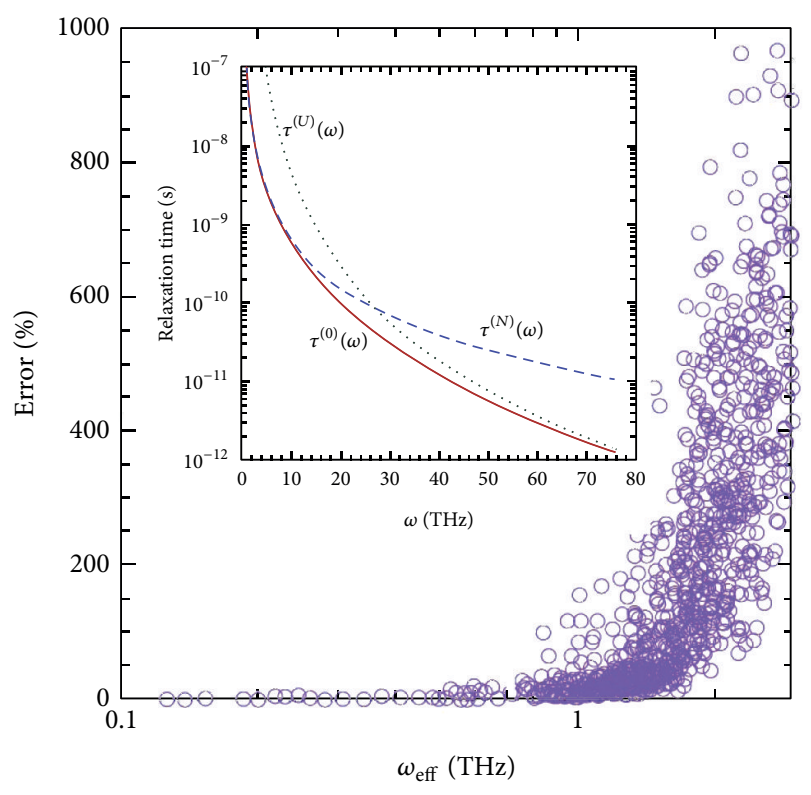

Figure 1: Percent error (color online) in $\left|V_{ \pm}^{(3)}\right|^{2}$ from the LWA compared to first principle for silicon at $300 \mathrm{~K}$. Insert shows the normal (blue dashed curve), Umklapp (green dotted curve), and total (red solid curve) relaxation times for the LA phonons calculated from Standard SMRTA by $a b$ initio approach. Adapted with permission from [110]. Copyrighted by the American Physical Society.

3.3.1. Intrinsic Phonon Scattering: Bulk Materials. Without any fitting parameters, Standard SMRTA with ab initio approach can accurately predict spectral phonon relaxation times and thermal conductivities. Ward and Broido [110] checked the validity of some old approximations introduced in Section 2: (1) long-wave approximation for three-phonon scattering and (2) ignoring optical phonons, using silicon and germanium as examples. First, the values of matrix element $\left|V_{ \pm}^{(3)}\right|^{2}$, which govern the scattering strength $\Gamma$, from $a b$ initio calculation for acoustic phonons are compared to those given by LWA. The percentage error of $\left|V_{ \pm}^{(3)}\right|^{2}$ is shown in Figure 1. We note that the LWA only works for the very low frequency $\omega_{\text {eff }}<0.8 \mathrm{THz}$, while, for most part $0.8<$ $\omega_{\text {eff }}<12 \mathrm{THz}$, the LWA gives large discrepancy, where $\omega_{\text {eff }} \equiv\left(\omega_{\lambda} \omega_{\lambda^{\prime}} \omega_{\lambda^{\prime \prime}}\right)^{1 / 3}$ is the geometric average of the threephonon frequencies. Second, the relaxation times of optical modes are found to only contribute less than $10 \%$ to the total thermal conductivity of silicon. However, ignoring optical modes is erroneous since the optical phonons are essential to provide channels for acoustic phonon scattering. The explicit calculation of millions of three-phonon scattering shows that optical phonons are involved in $50-60 \%$ of the total acoustic phonon-scattering processes in $\mathrm{Si}$ and Ge. Last, beyond the $\omega, T$ dependencies of $\tau$ listed in Table 1, which rely on many approximations, the ALD calculation can give more precise $\tau \omega, T$ dependence. This is illustrated in the inset of Figure 1, the relaxation times of the LA phonons in $\mathrm{Si}$ for $T=300 \mathrm{~K}$. By decomposing the total scattering into $U$ process and $N$ process, we find the $U$ process has a stronger frequency 
dependence $\tau^{(U)}(\omega) \sim \omega^{-4}$ than $N$ process $\tau^{(N)}(\omega) \sim \omega^{-2}$. The results also show that normal scattering governs the total relaxation time $\tau^{(0)}(\omega)$ at low frequency, while Umklapp scattering dominates at high frequency. Such $\sim \omega^{-4}$ relation is not expected in the analytical models in Table 1.

One flaw of the Standard SMRTA is that it does not grasp the interplay between the $N$ process and $U$ process. The right hand side of (15) can be decomposed as $\Gamma_{\lambda}^{(N)}+$ $\Gamma_{\lambda}^{(U)}$ (only consider intrinsic phonon scattering), according to whether they are $N$ or $U$ scattering events. The Standard SMRTA scheme treats the $N$ process and $U$ process as two independent scattering events and use Matthiessen's rule to account for the total relaxation time $1 / \tau_{\lambda}^{0}=1 / \tau_{\lambda}^{(N)}+1 / \tau_{\lambda}^{(U)}$, where $\tau_{\lambda}^{(N, U)}$ is defined as $\tau_{\lambda}^{(N, U)} \equiv 1 / \Gamma_{\lambda}^{(N, U)}$. However, it is well know that $N$ process does not contribute to thermal resistance directly. Instead, it affects the $U$ process (lowfrequency $N$ scattering produces high-frequency phonons which boosts $U$ process), and then the $U$ process produces thermal resistance. This error can be remedied in the Iterative Scheme by doing the iteration in (17). Therefore, the Standard SMRTA scheme only works for the system, where $U$ process dominates so that the $N$ scattering makes little difference to $U$ process as well as to thermal resistance $[51,110]$.

For $\mathrm{Si}$ and $\mathrm{Ge}$ at room temperature where the $U$ process is strong, the thermal conductivity predicted by Standard SMRTA scheme is only $5-10 \%$ smaller than that by Iterative Scheme [110], the latter shows excellent agreement with experiment (see Figure 1 of [61]).

In contrast, the $U$ scattering in diamond is much weaker [114-116] due to the much smaller phase space [117]. As a result, the thermal conductivity given by these two methods can differ by $50 \%$ at room temperature [110]. As shown in Figure 2, this discrepancy increases with decreasing temperature since the Umklapp scattering is weakened when temperature decreases. The thermal conductivity of diamond predicted by Iterative Scheme with $a b$ initio approach agrees excellently with experiment as shown in [110]. It is also noted that the Standard SMRTA scheme always underpredicts the thermal conductivity because it treats $N$ process as an independent channel for thermal resistance. On the other hand, if the relaxation time for $U$ process only is used in the calculation, the thermal conductivity is always overpredicted. This again confirms that the $N$ process has an indirect and partial contribution to the thermal resistance.

One important application of ALD calculation is to predict and understand the thermal conductivity of thermoelectric materials and help to design higher thermoelectric performance structures. Based on first principle calculation, Shiga et al. [104] obtain the frequency-dependent relaxation times of pristine PbTe bulk at $300 \mathrm{~K}$ as shown in Figure 3. At low-frequency region, TA phonons have longer relaxation times than LA phonons with $\tau$ 's exhibiting $\sim \omega^{-2}$ dependence. Separating the scattering rates into those of normal and Umklapp processes, they find the relations $\tau_{\text {Normal }} \sim \omega^{-2}$ and $\tau_{\text {Umklapp }} \sim \omega^{-3}$, which again indicate that the normal process dominates low-frequency region while the Umklapp dominates high-frequency part. By further studying the

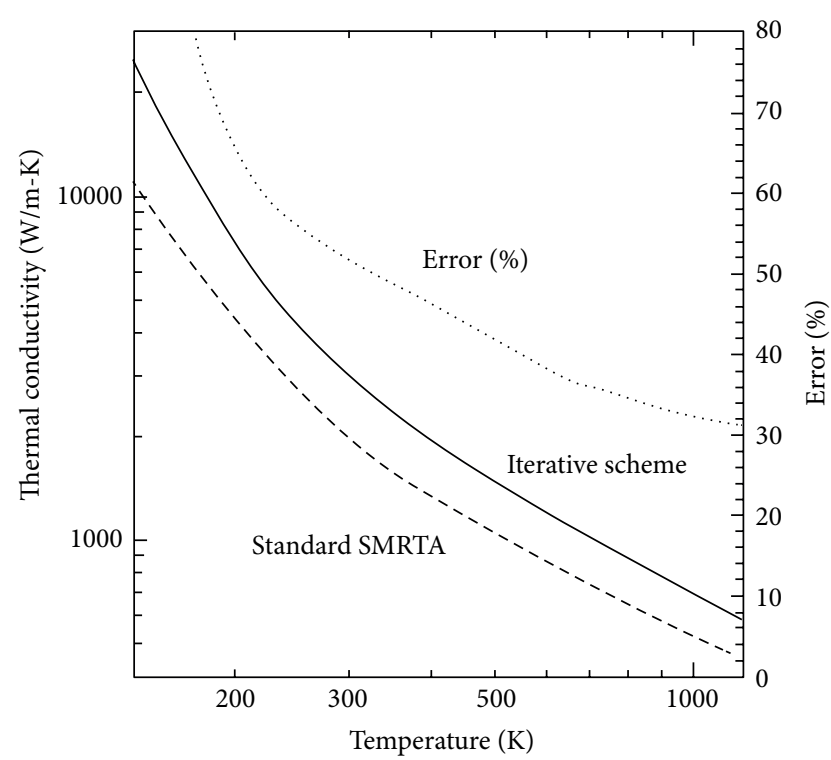

FIgURE 2: The calculated intrinsic lattice thermal conductivity of diamond for the Standard SMRTA (dashed line) and the Iterative Scheme (solid line), both by ab initio approach. Dotted line shows percent error of the Standard SMRTA result compared to the Iterative Scheme solution. Reprinted with permission from [51]. Copyrighted by the American Physical Society.

participation of each phonon mode to the total scattering rates, they find that the low thermal conductivity of $\mathrm{PbTe}$ is attributed to the strong scattering of LA phonons by TO phonons and the small group velocity of TA phonons. Figure 4 compares phonon relaxation times of $\mathrm{PbTe}$ and $\mathrm{PbSe}$ [106]. Although the anharmonicity of PbSe is normally expected to be larger due to the larger average Gruneisen parameter reported from experiments [121], in this work, it is found that, for TA mode, the relaxation times of $\mathrm{PbSe}$ are substantially longer than those of $\mathrm{PbTe}$. Surprisingly, the optical phonons are found to contribute as much as $25 \%$ for $\mathrm{PbSe}$ and $22 \%$ for $\mathrm{PbTe}$ to the total thermal conductivity at the temperature range 300-700 K. Motivated by the question that phonons with what kind of MFP contribute the most to the total thermal conductivity, the cumulative $k$ 's as functions of phonon MFP are calculated by ALD method with first principle approach as shown in Figure 5. Silicon is found to have phonon MFPs which span 6 orders of magnitude $\left(0-10^{6} \mathrm{~nm}\right)$, while the thermal transport in diamond is dominated by the phonon with narrow range of MFP (0.4$2 \mu \mathrm{m})$. It is found that the phonons with MFP below $4 \mu \mathrm{m}$ for silicon, $1.6 \mu \mathrm{m}$ for GaAs, $120 \mathrm{~nm}$ for $\mathrm{ZrCoSb}, 20 \mathrm{~nm}$ for $\mathrm{PbSe}$, and $10 \mathrm{~nm}$ for $\mathrm{PbTe}$ contribute $80 \%$ of total thermal conductivity. GaAs/AlAs superlattice is found to have similar phonon MFP with bulk GaAs. The curves of the alloy $\mathrm{Mg}_{2} \mathrm{Si}_{0.6} \mathrm{Sn}_{0.4}$ and its pure phases $\mathrm{Mg}_{2} \mathrm{Si}$ and $\mathrm{Mg}_{2} \mathrm{Sn}$ cross at the intermediate MFPs. These results provide great guidance for experimental works. For example, the PbTe-PbSe alloys with size of nanoparticle below $10 \mathrm{~nm}$ are synthesized and found to lead to as much as $60 \%$ reduction to the thermal 


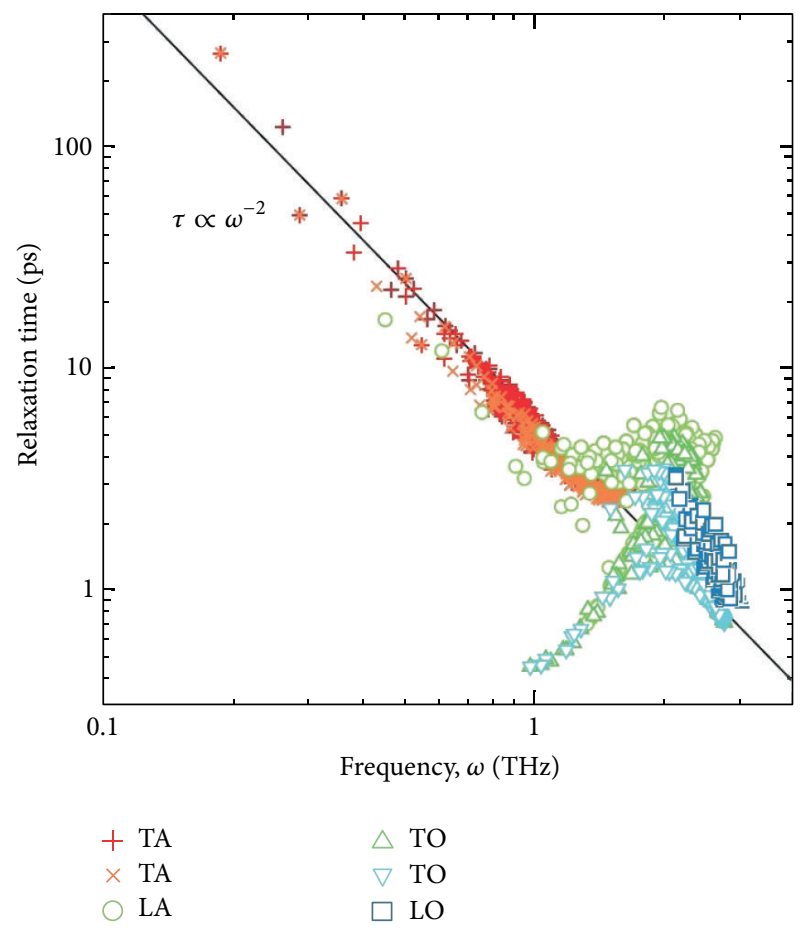

(a)

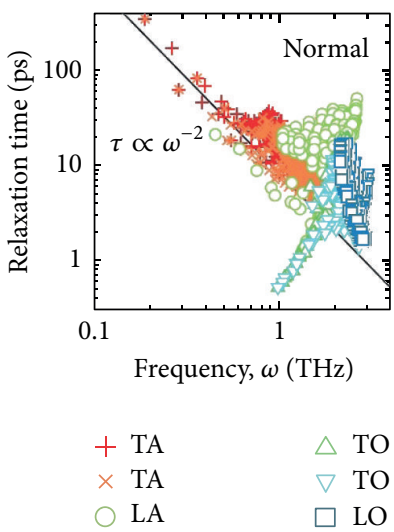

(b)

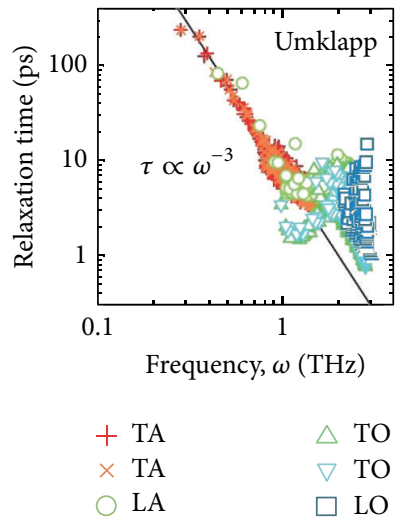

(c)

FIgURE 3: (a) Spectral phonon relaxation times of pristine PbTe bulk at $300 \mathrm{~K}$ by Standard SMRTA scheme with first principle IFCs. Relaxation times of (b) normal and (c) Umklapp processes, respectively. The solid lines plot (a) $\tau=6 \times 10^{12} \omega^{-2}$, (b) $\tau=8 \times 10^{12} \omega^{-2}$, and (c) $\tau=8 \times 10^{24} \omega^{-3}$. Reprinted with permission from [104]. Copyrighted by the American Physical Society.

conductivity which provides large space for improving ZT [122].

3.3.2. Single and Few-Layer 2D Materials, Nanoribbons, and Nanotubes. For single- and multilayer 2D materials, the boundary scattering from the sides perpendicular to the transport direction is much weaker than for 3D systems [123], making the boundary scattering expression in Table 1 unsuitable. Instead, when studying single-/multilayer graphene (SLG/MLG) and graphite [54, 55], single-wall carbon nanotubes (SWCNTs) [52, 53], single-/multilayer boron nitride (SLBN/MLBN), and boron nitride nanotubes (BNNTs) [56, 58], Lindsay and Broido only consider the boundary scattering from the two ends in the transport direction and show that

$$
\frac{1}{\tau^{\mathrm{bs}}}=\frac{2\left|\mathbf{v}_{\lambda} \cdot \widehat{z}\right|}{L}
$$

works well in accounting for the boundary scattering, with $L$ being the length between boundaries in the transport direction $\widehat{z}$. Such formula has been shown to give correct thermal conductivity values of nanotubes [124] and nanoribbons [125] in the ballistic limit $(L \rightarrow 0)$ and diffusive limit $(L \rightarrow \infty)$.

Vibrations in 2D lattices are characterized by two types of phonons: those vibrating in the plane of layer (TA and LA) and those vibrating out of plane, so called flexural phonons (ZA and ZO). Lindsay et al. [54] find the selection rule for all orders in anharmonic phonon-phonon scattering in the 2D crystals: only even numbers (including zero) of flexural phonons can be involved, arising from the reflection symmetry perpendicular to the plane of layer. This selection rule has forbidden about $60 \%$ of both $N$ and $U$ threephonon scattering phase space of ZA phonons for single layer graphene. They show that such suppressed scattering yields long relaxation time and mean free path for $\mathrm{ZA}$ phonons, leading to ZA phonons contributing most of the thermal conductivity of SLG, about $70 \%$ at room temperature (another cause being the large density of states and occupation number of ZA modes). However, this conclusion is still under debate since this approach does not include the fourth- and higher-order phonon scattering rates, which are not necessarily low since the reflection symmetry allows more 4-phonon processes than 3-phonon processes. Actually, the method of spectral energy analysis based on MD (discussed in Section 4 ) indicates that only $25 \%-30 \%$ of the total $k$ is contributed by ZA mode at room temperature [126-128]. It should be noted that MD has its own drawback of not reproducing the Bose-Einstein distribution for graphene phonons at room temperature. Hence, the discrepancies between the two methods still need further study.

The selection rule mentioned above does not hold for multilayer graphene, twisted graphene, graphite (because of the interlayer coupling), CNT (due to the curvature), graphene nanoribbon (GNR) (due to boundary scattering), 


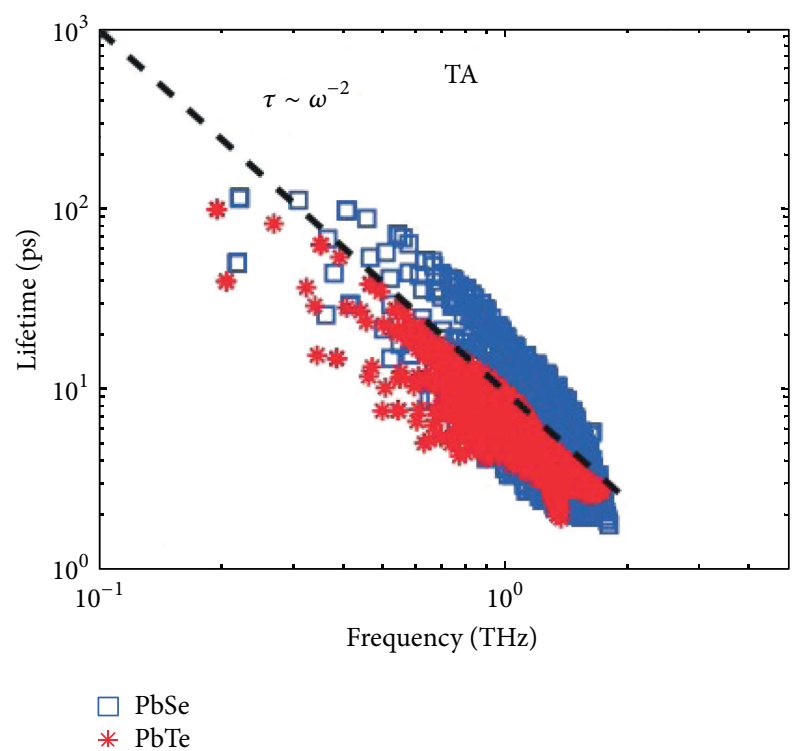

(a)

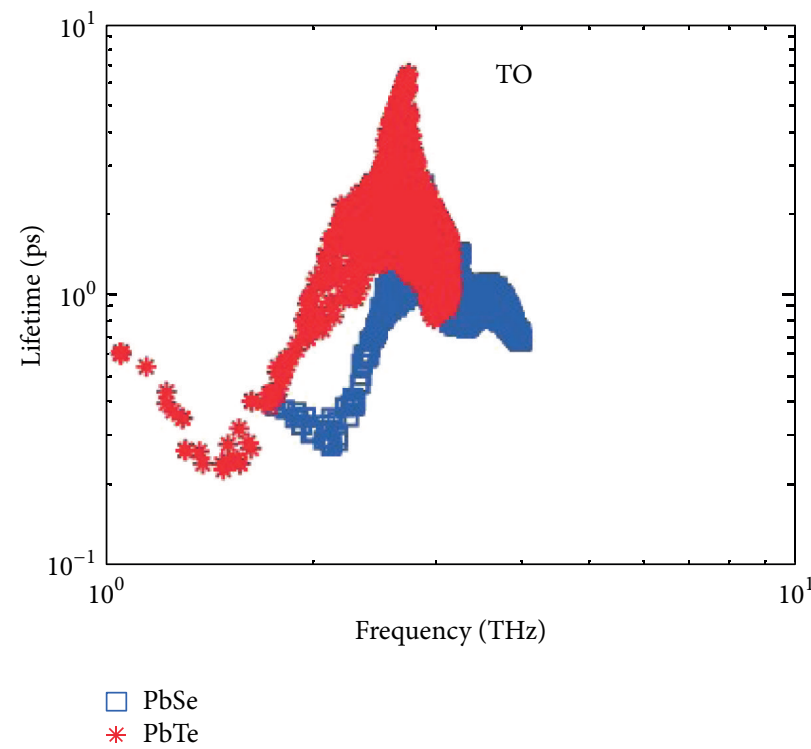

(c)

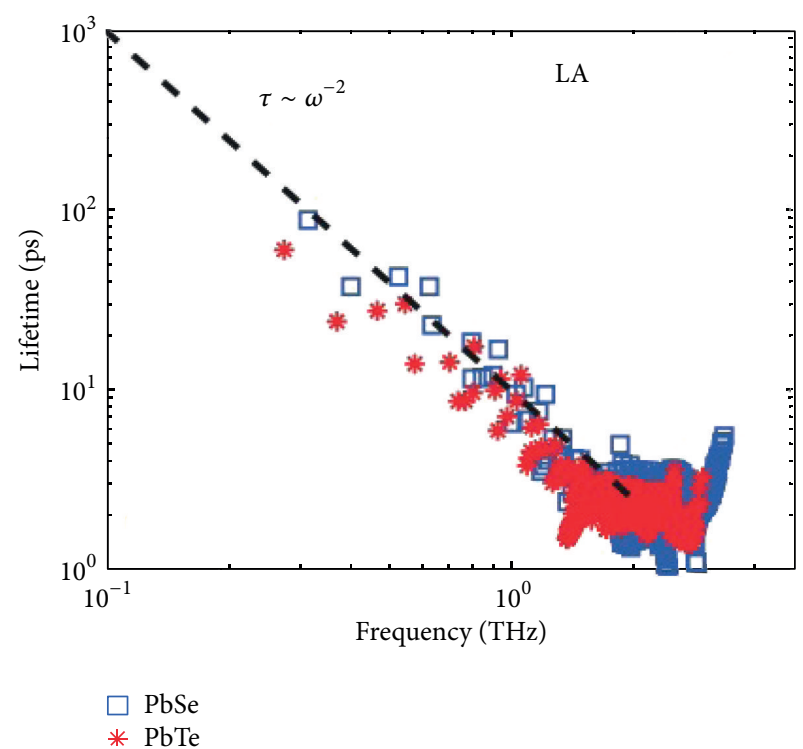

(b)

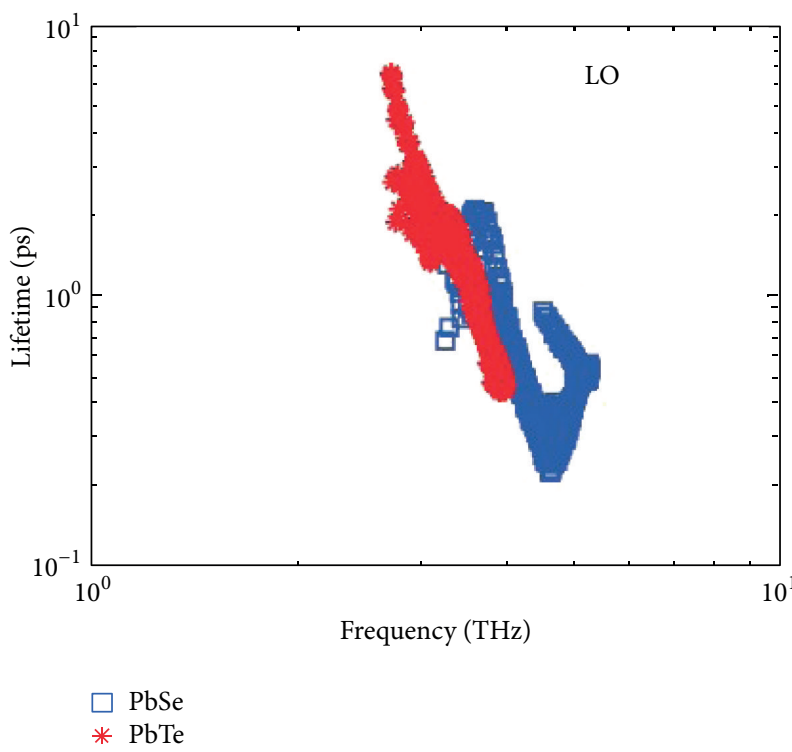

(d)

FIGURE 4: Spectral phonon relaxation times of PbSe bulk (squares) and PbTe bulk (crosses) at $300 \mathrm{~K}$ by Standard SMRTA scheme with IFCs from first principle calculation: (a) TA, (b) LA, (c) TO, and (d) LO. Reprinted with permission from [106]. Copyrighted by the American Physical Society.

substrate-supported graphene (due to scattering with the substrate), and defective graphene (due to defective scattering). Therefore, the thermal conductivity of these structures is typically lower than that of single layer graphene, and the contribution of each phonon mode changes [54, 129132]. In Figure 6, single-layer graphene, GNR, and SWCNT are compared, where graphene has an infinite width and finite length $L$, SWCNT has a finite diameter $d$ and length $L$, and GNR has a finite width $W=\pi d$ with artificial periodic boundary condition applied. As expected, $k_{\mathrm{RTA}}$ underpredicted thermal conductivity. SWCNT is found to have a lower thermal conductivity than graphene with a minimum value of $77 \%$ of $k_{\text {graphene }}$ at a critical diameter $d \approx$
$1.5 \mathrm{~nm}$. From this critical diameter, $k_{\mathrm{SWCNT}}$ increases with increasing diameter and reaches $90 \%$ of $k_{\text {graphene }}$ at $d \approx 4 \mathrm{~nm}$. On the other hand, if $d$ goes small enough, phonon-phonon scattering decreases and the thermal conductivity increases. At this short limit of $d$, the system becomes more like a 1D chain which generally has much larger thermal conductivity than $2 \mathrm{D}$ and $3 \mathrm{D}$ systems. The increasing trend of $k_{\mathrm{GNR}}$ with decreasing $d$ comes from the reason that the decrease of the width $\pi d$ pushes the optical modes to higher frequencies and thus the $U$ scattering by optical phonons becomes weaker.

For 2D materials and nanotube structures, the $N$ scattering is usually strong. For example, for CNT, Lindsay et al. [52] find that all the three-acoustic-phonon scatterings are $N$ 


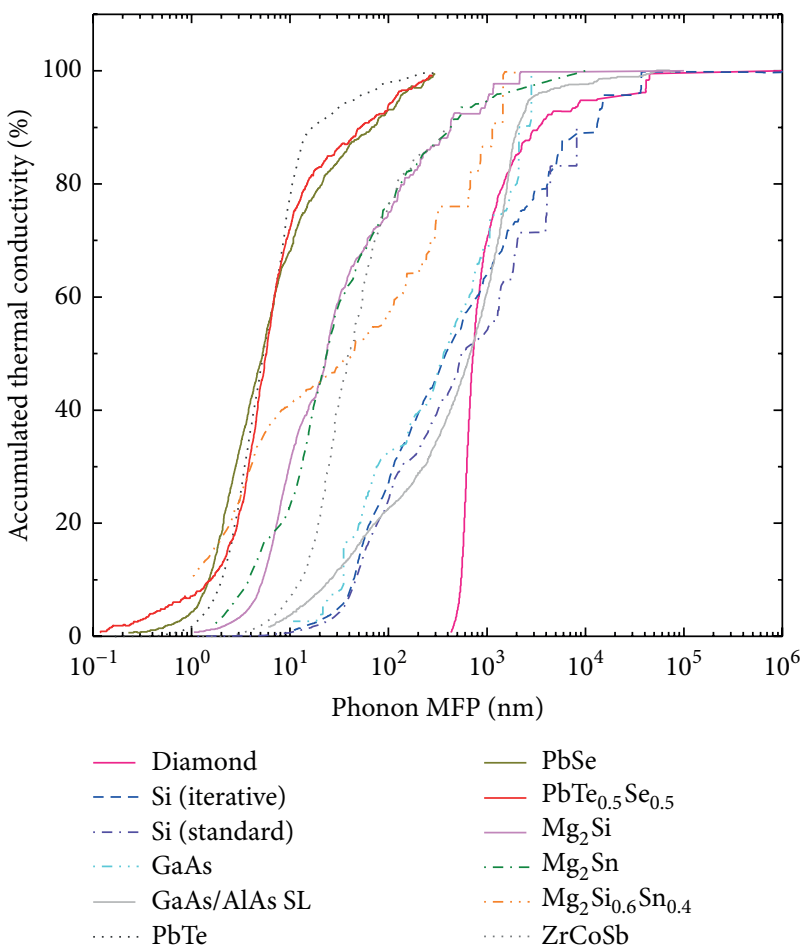

FIgURE 5: The accumulated thermal conductivity of different bulk materials as a function of phonon mean free path at room temperature calculated from ALD by first principle approach. (Diamond [64]; Si (iterative) [64]; Si (standard) [107]; GaAs [118]; FaAs/AlAs [119]; PbTe [106]; PbSe [106]; $\mathrm{PbTe}_{0.5} \mathrm{Se}_{0.5}$ [106]; $\mathrm{Mg}_{2} \mathrm{Si}$ [63]; $\mathrm{Mg}_{2} \mathrm{Sn}$ [63]; $\mathrm{Mg}_{2} \mathrm{Si}_{0.6} \mathrm{Sn}_{0.4}$ [63]; $\left.\mathrm{ZrCoSb}[120]\right)$.

processes, so that the $U$ process is, respectively, weak because it must involve optical phonons, which are less likely to be thermally excited. Thus, the Iterative Scheme can be used to layer and tube structure, rather than Standard SMRTA whose results are less accurate. Figure 7 shows the ratio between thermal conductivities $k_{L}$ (from Iterative Scheme) and $k_{\mathrm{RTA}}$ (from Standard SMRTA), where the discrepancy is typically larger than $100 \%$. The ZA mode shows the largest divergence which can reach 8 -fold at length of $10 \mu \mathrm{m}$, because the flexural phonons have lower frequencies than other modes and thus stronger $N$ process than $U$ process.

3.3.3. Boundary Scattering: Nanowires. For nanowires, the Casimir model (Table 1) has been applied to predict thermal conductivity in many works [24-27, 27-32, 133] recently. Generally, $k$ decreases with decreasing nanowire diameter; however, at some point, as the diameter continues to decrease, $k$ will increase due to the 3D-1D transition. The main problems are that the results strongly rely on fitting parameters and that the use of Matthiessen approximation is still questioned. Instead, Ziman [95] presents an approach of solving space-dependent BTE (Peierls-BTE [134]). The final result of this Peierls-BTE approach gives, according to the

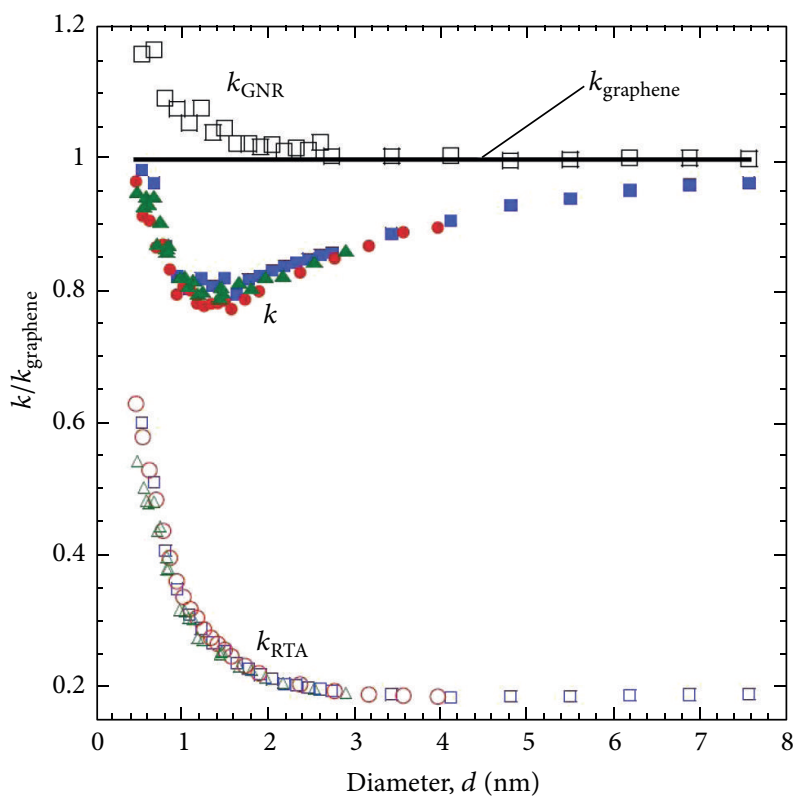

FIGURE 6: Thermal conductivity $k$ versus diameter $d$ (color online) for single wall carbon nanotubes. Solid red circles, blue squares, and green triangles represent zigzag, armchair, and chiral predicted by Iterative Scheme; open red circles, blue squares, and green triangles are those from Standard SMRTA. Black line shows $k_{\text {graphene }}$ while the black open squares give $k_{\mathrm{GNR}}$. For all cases, length $L=3 \mu \mathrm{m}$ and $T=300 \mathrm{~K}$. Reprinted with permission from [53]. Copyrighted by the American Physical Society.

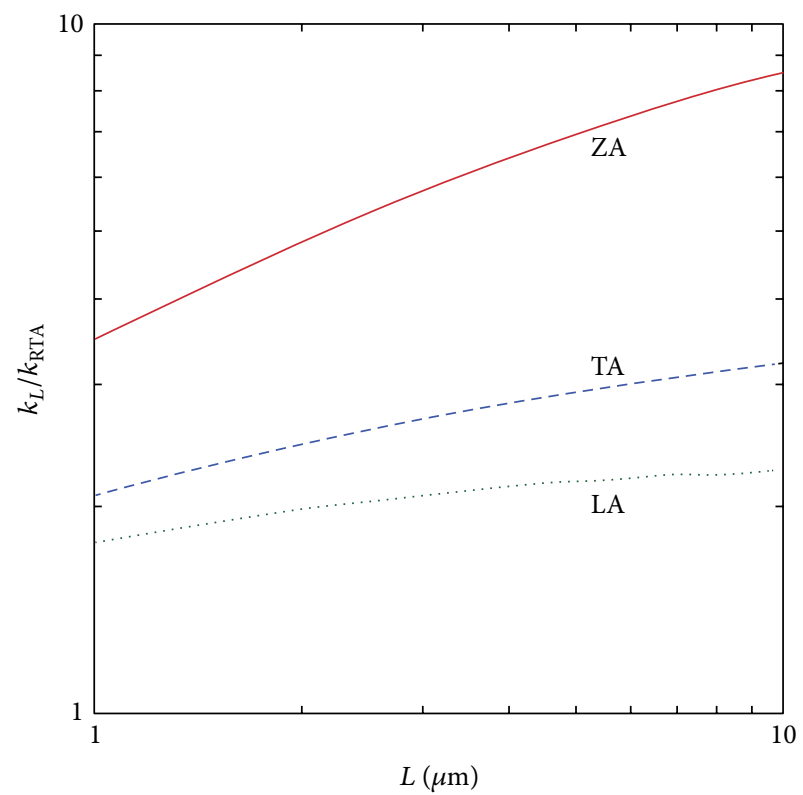

FIGURE 7: The ratio (color online) between thermal conductivity predicted from Iterative Scheme $\left(k_{L}\right)$ and Standard SMRTA $\left(k_{\text {RTA }}\right)$ of graphene as a function of length $L$ for temperature $300 \mathrm{~K}, k_{\mathrm{ZA}}$ (solid red), $k_{\mathrm{TA}}$ (blue dashed), and $k_{\mathrm{LA}}$ (green dotted) being the contributions from different branches. Reprinted with permission from [54]. Copyrighted by the American Physical Society. 


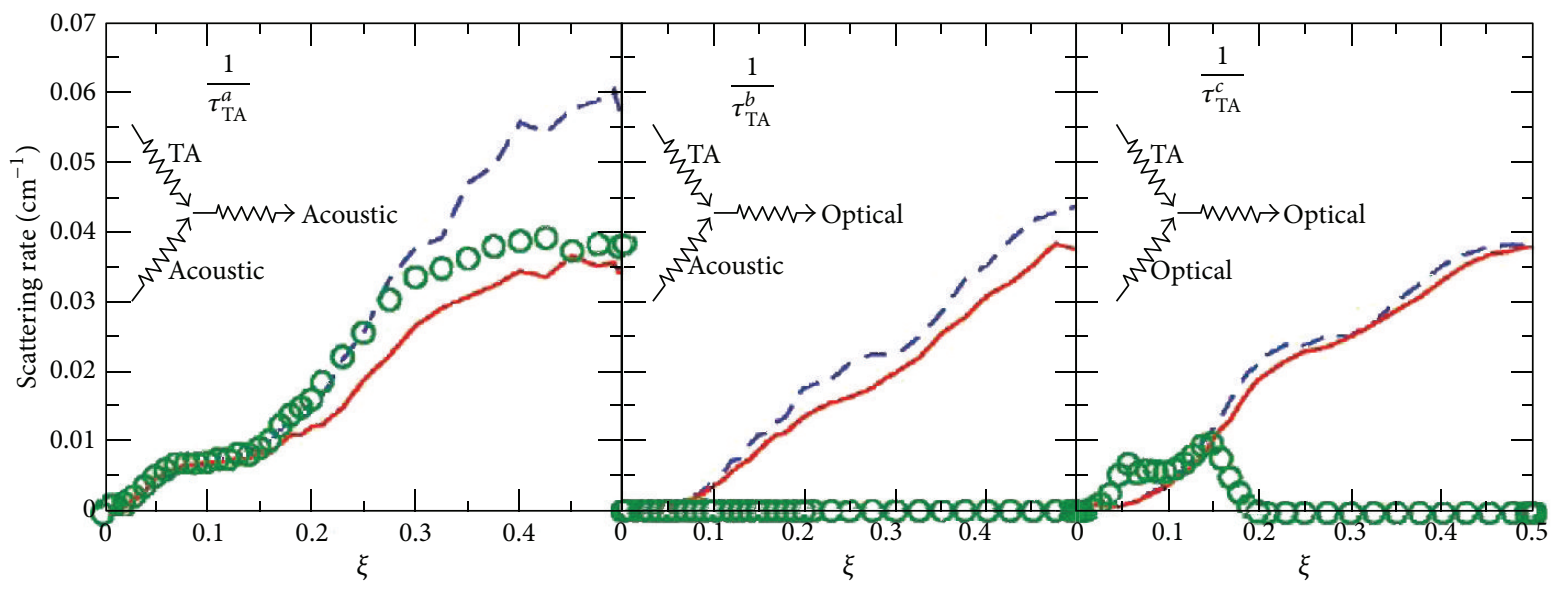

(a)

(b)

(c)

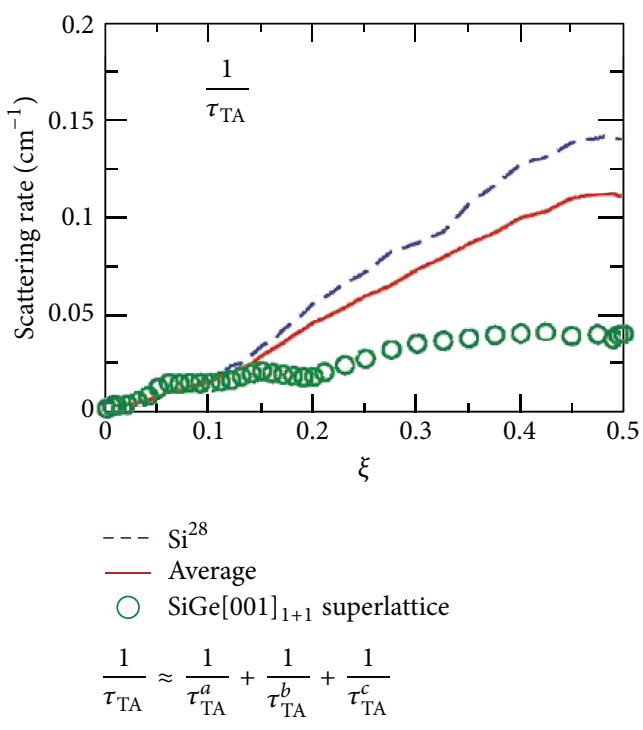

(d)

FIGURE 8: Scattering rate (color online) of TA mode at $300 \mathrm{~K}$ due to (a) absorption of an acoustic phonon to yield another acoustic phonon, (b) absorption of an acoustic phonon to yield an optical phonon, (c) absorption of an optical phonon to yield another optical phonon, and (d) total scattering rate, along $(\xi, \xi, \xi)$ in ${ }^{28} \mathrm{Si}$, "average material," and $\mathrm{SiGe}[001]_{1+1}$ superlattice. $\xi$ is reduced wave vector, with $\mathbf{k}=(\xi, \xi, \xi) \cdot 2 \pi / a$. Reprinted with permission from [108]. Copyright (2011) American Chemical Society.

simplification by Li et al. $[63,64]$, the position-dependent spectral phonon relaxation time

$$
\tau_{\mathbf{r}, \lambda}=\tau_{\lambda}^{0}\left(1+\bar{\Delta}_{\lambda}\right)\left\{1-e^{-\left|\left(\mathbf{r}-\mathbf{r}_{b}\right) / \tau_{\lambda}^{0} \nu_{\lambda}\right|} G_{\mathbf{r}, \lambda}\right\}
$$

where $\bar{\Delta}_{\lambda}$ is the average value of $\Delta_{\lambda}$ over the cross section, $\mathbf{r}_{b}$ is the point on the surface with $\mathbf{r}-\mathbf{r}_{b}$ being the same direction with group velocity vector $\mathbf{v}_{\lambda}$, and $G_{\mathbf{r}, \lambda}$ describes the boundary condition with $G_{\mathbf{r}, \lambda}=1$ for completely diffusive and $G_{\mathbf{r}, \lambda}=0$ for mirror like. The average of $\tau_{\mathbf{r}, \lambda}$ over cross section is

$$
\bar{\tau}_{\lambda}=\tau_{\lambda}^{0}\left(1+\bar{\Delta}_{\lambda}\right)\left[\frac{1}{S_{c}} \int_{S_{c}}\left\{1-e^{-\left|\left(\mathbf{r}-\mathbf{r}_{b}\right) / \tau_{\lambda}^{0} v_{\lambda}\right|} G_{\mathbf{r}, \lambda}\right\} d \mathbf{s}\right]
$$

So far, the calculation for nanowire still needs an adjustable parameter to account for the boundary scattering.

3.3.4. Impurity-Isotope Scattering: Doping and Alloys. From second-order perturbation theory $[34,95,135]$, assuming that the isotopes are distributed randomly, the single scattering rate by the isotopes $[82,136]$ is given by

$$
\Gamma_{\lambda \lambda^{\prime}}^{\text {iso }}=\frac{\pi}{2 N_{c}} \omega_{\lambda} \omega_{\lambda^{\prime}} \sum_{b}^{n} g_{b}\left|\mathbf{e}_{\lambda}^{b} \cdot \mathbf{e}_{\lambda^{\prime}}^{b *}\right|^{2} \delta\left(\omega_{\lambda}-\omega_{\lambda^{\prime}}\right)
$$

where $N_{c}$ and $n$ stand for the number of unit cells and the number of atoms per unit cell, respectively, $\mathbf{e}_{\lambda}^{b}$ is the 


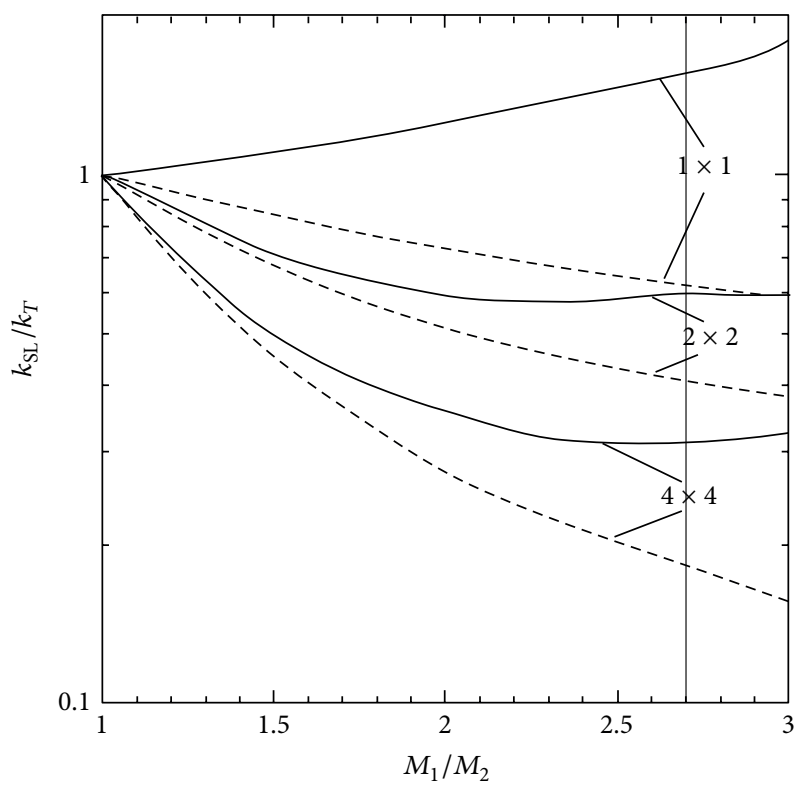

FIgURE 9: Thermal conductivities calculated by Standard SMRTA method (dashed lines) and Iterative Scheme (solid lines) of $1+1,2+2$, and $4+4 \mathrm{Si} / \mathrm{Ge}$-based SLs as a function of $M_{1} / M_{2}$, the mass ratio of constituent atoms. The thin vertical line shows the Ge/Si mass ratio. Reprinted with permission from [50]. Copyrighted by the American Physical Society.

eigenvector of $\lambda$ mode in the basis atom $b$ part, $*$ denotes complex conjugate, and

$$
g_{b}=\sum_{i} f_{i b}\left(1-\frac{m_{i b}}{\bar{m}_{b}}\right)^{2}
$$

characterizes the magnitude of mass disorder, where $i$ indicates isotope types, $f_{i b}$ is the fraction of isotope $i$ in lattice sites of basis atom $b, m_{i b}$ is the mass of isotope $i$, and $\bar{m}_{b}$ is the average atom mass of basis $b$ sites. Sum over all the modes $\lambda^{\prime}$ with $\lambda^{\prime} \neq \lambda$, the total scattering rate, or the inverse relaxation time of mode $\lambda$ is

$$
\frac{1}{\tau_{\lambda}^{\text {iso }}}=\sum_{\lambda^{\prime}} \Gamma_{\lambda \lambda^{\prime}}^{\text {iso }}=\frac{\pi}{2 N_{c}} \omega_{\lambda}^{2} \sum_{b}^{n} \sum_{\lambda^{\prime}} g_{b}\left|\mathbf{e}_{\lambda}^{b} \cdot \mathbf{e}_{\lambda^{\prime}}^{b *}\right|^{2} \delta\left(\omega_{\lambda}-\omega_{\lambda^{\prime}}\right) .
$$

For cubic symmetry system [82] such as $\mathrm{Si}, \mathrm{Ge}$, and Ar, the summation of eigenvectors in (26) can be reduced to

$$
\begin{aligned}
\frac{1}{\tau_{\lambda}^{\text {iso }}} & =\frac{\pi}{6 n N_{c}} g \omega_{\lambda}^{2} \sum_{\lambda^{\prime}} \delta\left(\omega_{\lambda}-\omega_{\lambda^{\prime}}\right) \\
& =\frac{\pi V_{0}}{6} g \omega^{2} \bar{D}(\omega) \\
& =\frac{\pi}{2} g \omega^{2} D(\omega),
\end{aligned}
$$

where $g$ is given by (5), $V_{0}=V /\left(n N_{c}\right)$ is the volume per atom, $\bar{D}(\omega)=\sum_{\lambda^{\prime}} \delta\left(\omega_{\lambda}-\omega_{\lambda^{\prime}}\right) / V$ is density of states per unit volume, and $D(\omega)$ is density of states normalized to unity, noticing that the total amount of states is $3 n N_{c}$ and the total volume

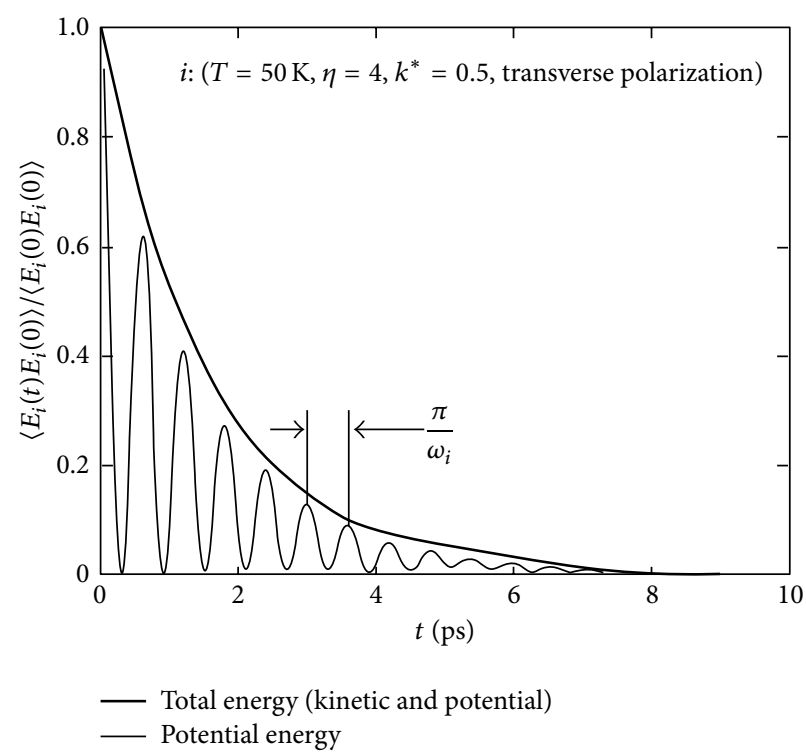

FIGURE 10: Autocorrelation functions of potential and total energies of time-dependent normal modes of TA mode at $k^{*}=0.5$ in $[1,0,0]$ direction of argon at $50 \mathrm{~K}$. Reprinted with permission from [67]. Copyrighted by the American Physical Society.

is $V=V_{0} n N_{c}$. Rewriting the $D(\omega)$ [82], one can obtain the formula in Table 1. From (27), the relaxation time of mode $\lambda$ only depends on the frequency rather than the phonon branches.

Equation (24) or (26) combined with the Standard SMRTA or Iterative Scheme have been used to predict the spectral phonon relaxation times of doped materials and even alloys. For isotope-doped system, this method can be directly applied, such as silicon and germanium $[47,60$, 91], hexagonal boron nitride layers and nanotube $[56,58]$, $\mathrm{GaN}$ [57], and $\mathrm{SiC}$ [48]. For alloy, the disordered crystal is treated as an ordered one of the average atomic mass, lattice parameter, and force constants. This approach, the so-called virtual crystal approach, first introduced by Abeles [137], has been applied to Si-Ge alloys $[65,109]$ and $\mathrm{PbTe}_{(1-\mathrm{x})} \mathrm{Se}_{\mathrm{x}}$ alloys [106] by ab initio IFCs, $\left(\mathrm{Bi}_{(1-\mathrm{x})} \mathrm{Sb}_{\mathrm{x}}\right)_{2} \mathrm{Te}_{3}$ alloys [138] with classical potential, and $\mathrm{Ni}_{0.55} \mathrm{Pd}_{0.45}$ alloys [139] for comparison with experiment. It turns out that the secondorder perturbation $((24)$ or (26)) can give good prediction even for large mass disorder.

3.3.5. Superlattices. Superlattices (SLs), composed of periodically arranged layers of two or more materials, have been extensively investigated in the aspect of thermal transport. Because of the heat transport suppression by interfaces and mass mismatch, superlattice has been designed to have a lower thermal conductivity than pure bulk. SLs are classified into two categories: diffuse and specular interfaces. The phonons in the first case are diffusively scattered by interfaces, while the phonons in the latter one propagate through the whole structure as if in one material, so-called coherent phonon transport [119]. Although proposed in theoretical studies long ago, the coherent phonon transport in SLs was 


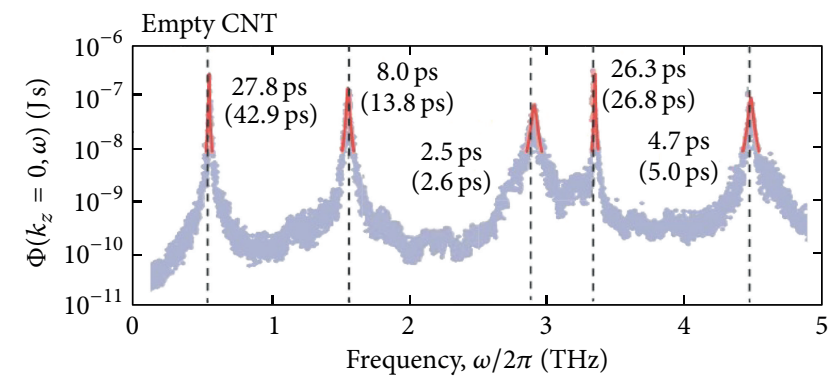

(a)

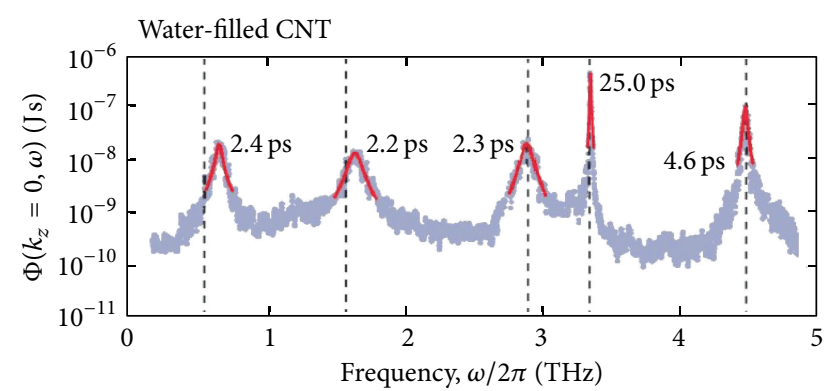

(b)

FIGURE 11: SED functions (color online) of (a) empty CNT and (b) water-filled CNT along $k_{z}=0$ for frequencies below $5 \mathrm{THz}$ at $T=298 \mathrm{~K}$. Reprinted with permission from [77]. Copyrighted by the American Physical Society.

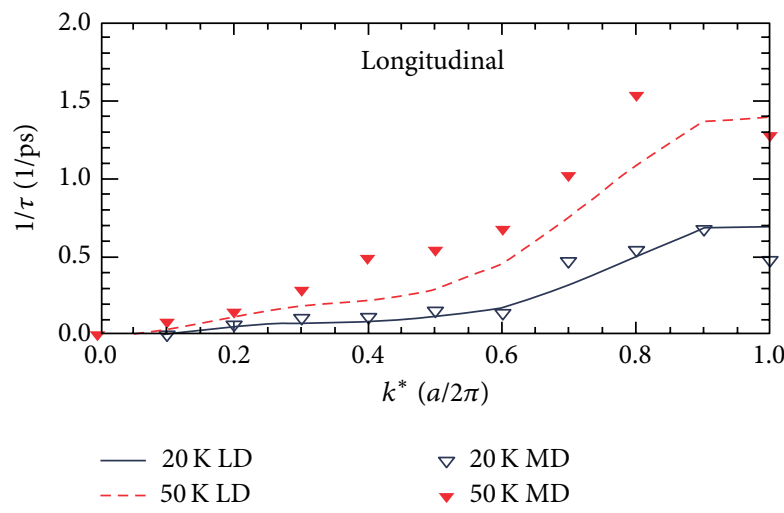

(a)

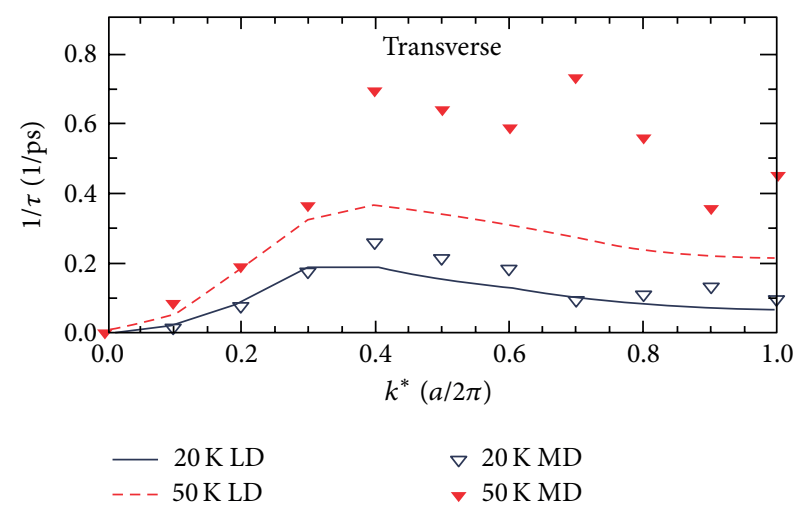

(b)

FIGURE 12: The inverse relaxation times (color online) of LA and TA mode of argon as functions of wave vector in [109] direction, predicted by Standard SMRTA method at $20 \mathrm{~K}$ (solid curve) and $50 \mathrm{~K}$ (dashed curve) and time domain NMA at $20 \mathrm{~K}$ (open triangle) and $50 \mathrm{~K}$ (solid triangle). Adapted with permission from [102]. Copyrighted by the American Physical Society.

not observed in experiments until Luckyanova et al. studied finite-thickness GaAs/AlAs SLs by time-domain thermoreflectance measurements [119]. It is found that the cross-plane $k$ increases linearly with the number of periods when keeping the periods constants. Such phenomenon suggests that the phonon MFPs are equal to the sample thickness and the phonons do not "see" interfaces. Luckyanova et al. performed a first principle calculation (Standard SMRTA scheme) of GaAs/AlAs SLs to support their experimental results. They found that the anharmonic scattering rates and interface scattering rates, within the low-frequency region, had the frequency dependence as $\sim \omega^{-2}$ and $\sim \omega^{-4}$, respectively. The high-frequency phonons are scattered by interfaces, while the low-frequency phonons have long MFPs and thus can propagate though the entire SLs. Another evidence that the phonons in SL do not "see" interfaces is the fact that the accumulated $k$ of GaAs/ALAs SL is similar to bulk GaAs as shown in Figure 5. All the calculated results support the experimental finding of coherent phonon propagations. In the following discussion of SLs, we only consider coherent phonon transport.

Generally, $k$ increases with increasing period length $P_{L}$ (at the limit $P_{L} \rightarrow \infty k$ increases to that of the pure bulk material). However, it is found that, for extremely short period length, $k$ even increases with decreasing $P_{L}$. This leads to a phenomenon that $k$ as a function of $P_{L}$ reaches its minimum at a critical $P_{L}$, and calculation of such value of $P_{L}$ is crucially important for designing low thermal conductivity materials. For instance, Yang et al. found that the isotope silicon superlattice ${ }^{\text {iso }} \mathrm{Si} /{ }^{28} \mathrm{Si}$ nanowire had its lowest $k$ at $P_{L} \approx 1 \mathrm{~nm}$ [6]; Hu and Poulikakos noticed that the Si/Ge superlattice nanowire with $3.07 \mathrm{~nm}$ diameter had its lowest $k$ at $P_{L} \approx 4 \mathrm{~nm}$ [7]; $k$ of GaAs/AlAs superlattice [8] as a function of periodic length also obeys this principle. The exact phonon relaxation time explanation for such phenomenon is not available until the ALD method is explored [50, 59, 101, 103, 108].

Garg et al. [108] studied short-period $(0.3 \mathrm{~nm})$ $\mathrm{Si} / \mathrm{Ge}[001]_{1+1}$ superlattice using Standard SMRTA with $a b$ initio IFCs. They find that the thermal conductivity and phonon relaxation time of such superlattice are even greater than those of the two composition materials: pristine $\mathrm{Si}$ and Ge bulks. To understand this unusual behavior, the inverse relaxation time of TA mode is calculated and shown in Figure 8. Also plotted are the detailed three-phonon scattering rates for (a) $\mathrm{TA}+\mathrm{A} \rightarrow \mathrm{A}$, (b) $\mathrm{TA}+\mathrm{A} \rightarrow \mathrm{O}$, and 


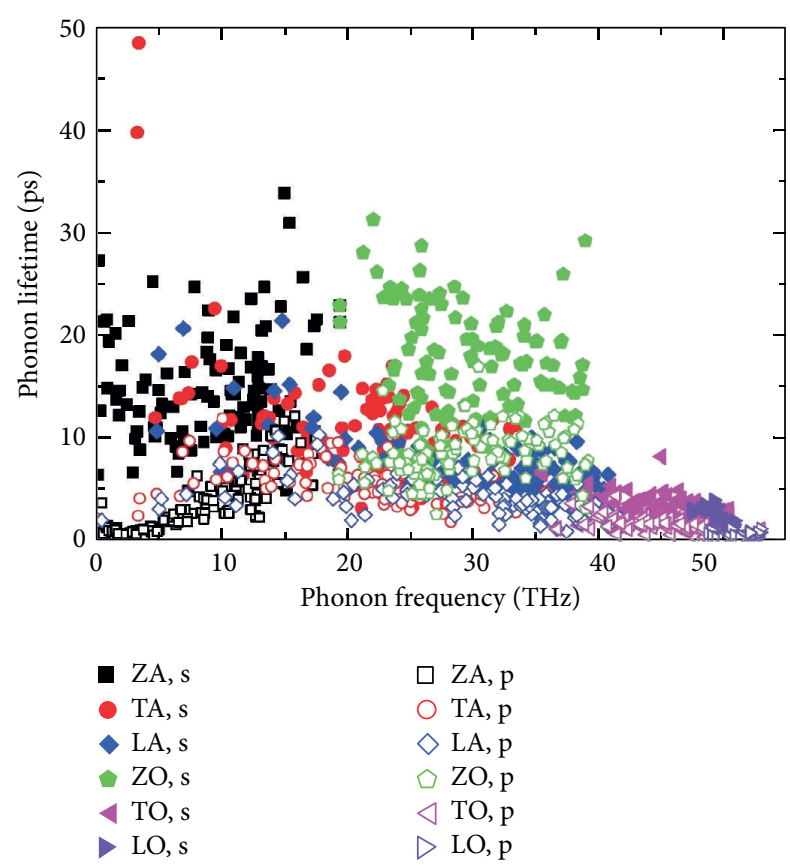

FIGURE 13: The relaxation time (color online) of suspended (" $\mathrm{s}$ ") and $\mathrm{SiO}_{2}$ supported ("p") single-layer graphene as a function of phonon frequency for different phonon branches at temperature 300 K. Reprinted with permission from [140]. Copyright 2012, AIP Publishing LLC.

(c) $\mathrm{TA}+\mathrm{O} \rightarrow \mathrm{O}$, where $\mathrm{A}$ and $\mathrm{O}$ stand for acoustic and optical, respectively. The "average material" is an imaginary material with averaged mass and potential of $\mathrm{Si}$ and $\mathrm{Ge}$ Bulk, for comparison with SiGe[001 $]_{1+1}$ superlattice. We note that only the (a) component can provide scattering for TA phonons, and that both (b) and (c) which affiliate with optical modes are almost completely absent. This indicates that the gap between optical and acoustic modes becomes so larger that the acoustic phonon can hardly be scattered by optical phonons. Such reduced scattering makes the relaxation times and thermal conductivity much larger than the two composition bulk materials.

More generally, Broido and Reinecke [59] and Ward and Broido [50] studied $M_{1} / M_{2[n+n]}$ superlattice (the diamond structure with periodical $n$ layers of mass $M_{1}$ atoms and mass $M_{2}$ atoms in $[0,0,1]$ direction) using Iterative Scheme. In these two works, the IFCs are determined using Keating model $[144,145]$ and adiabatic bond charge $(A B C)$ model $[146,147]$, respectively. In such $M_{1} / M_{2[n+n]}$ superlattice, the high thermal conductivity is also found for $n=1$ (Figure 9). When $n$ and mass ratio $M_{1} / M_{2}$ are increasing, $k$ is determined by the competition between the decease of phonon group velocity and the increase of phonon relaxation time. It turns out that, from about $M_{1} / M_{2}=2.3$, the latter competitor dominates and thus $k$ increase with increasing mass ratio. In Figure 9, as expected the $k$ 's from Iterative Scheme are generally larger than those from Standard SMRTA method. This difference increases with increasing mass ratio because the occurrence of $N$ process increases when the acousticoptical gap gets larger.

\section{MD Simulation}

4.1. Time Domain Normal Mode Analysis. The time domain normal mode analysis based on MD simulation was first proposed by Ladd et al. [66] and then modified by McGaughey and Kaviany [67]. From (10), a result of SMRTA, the relaxation time $\tau_{\lambda}$ can be obtained by

$$
\tau_{\lambda}=\frac{\int_{0}^{\infty}\left\langle n_{\lambda}^{\prime}(t) n_{\lambda}^{\prime}(0)\right\rangle d t}{\left\langle n_{\lambda}^{\prime 2}(0)\right\rangle} .
$$

According to the analysis by Ladd et al. [66], the fluctuation $n_{\lambda}^{\prime}$ in (29) can be replaced by the total phonon occupation number $n_{\lambda}$, which does not influence the calculation of thermal conductivity when considering that the ensembleaverage heat current is zero. From lattice dynamics [34, 148], the occupation number $n_{\lambda}$ is proportional to the energy of single phonon mode $\lambda$ described by the normal mode amplitude:

$$
E_{\lambda}=\frac{1}{2}\left(\dot{q}_{\lambda} \dot{q}_{\lambda}^{*}+\omega_{\lambda}^{2} q_{\lambda} q_{\lambda}^{*}\right)
$$

where $q_{\lambda}$ is the normal mode coordinate. Thus, (29) is transformed to

$$
\tau_{\lambda}=\frac{\int_{0}^{\infty}\left\langle E_{\lambda}(t) E_{\lambda}(0)\right\rangle d t}{\left\langle E_{\lambda}^{2}(0)\right\rangle},
$$

which is exactly what McGaughey and Kaviany [67] got, with the equivalent form $\left\langle E_{\lambda}(t) E_{\lambda}(0)\right\rangle /\left\langle E_{\lambda}^{2}(0)\right\rangle=\exp \left(-t / \tau_{\lambda}\right)$. Originally, Ladd et al. [66] only considered the potential energy and assumed $E_{\lambda} \sim q_{\lambda} q_{\lambda}^{*}$, which does not influence the result since the normal mode has the form $[66,149]$

$$
q_{\lambda}(t)=q_{\lambda, 0} \exp \left[i\left(\omega_{\lambda}^{A}+i \eta_{\lambda}\right) t\right],
$$

where $q_{\lambda, 0}$ is the vibration amplitude, a constant for a given mode $\lambda, \omega_{\lambda}^{A}=\omega_{\lambda}+\Delta_{\lambda}$ is the anharmonic frequency, and $\eta_{\lambda}$ is linewidth. With the help of this equation, both $E_{\lambda} \sim q_{\lambda} q_{\lambda}^{*}$ and $E_{\lambda} \sim \dot{q}_{\lambda} \dot{q}_{\lambda}^{*}$ give the equivalent value of $\tau_{\lambda}=1 / 2 \eta_{\lambda}$.

The calculation of normal mode coordinate $q_{\lambda}(t)$ is required to evaluate $E_{\lambda}$ in (30) and further predict $\tau_{\lambda}$ in (31). From lattice dynamics [148],

$$
\begin{aligned}
q_{\lambda}(t) & =\sum_{\alpha}^{3} \sum_{b}^{n} \sum_{l}^{N_{c}} \sqrt{\frac{m_{b}}{N_{c}}} u_{\alpha}^{l, b}(t) e_{b, \alpha}^{\lambda *} \exp \left[i \mathbf{k} \cdot \mathbf{r}_{0}^{l}\right] \\
& =\sum_{\alpha}^{3} \sum_{b}^{n} e_{b, \alpha}^{\lambda *} q_{\alpha}^{b}(\mathbf{k}, t),
\end{aligned}
$$

where $\alpha$ indicates $x, y, z$ directions, $u_{\alpha}^{l, b}(t)$ is $\alpha$ component of the displacement of the $b$ th atom in $l$ th unit cell from its equilibrium position, $\mathbf{r}_{0}^{l}$ is the equilibrium position of unit cell $l$, the star denotes complex conjugate, and $q_{\alpha}^{b}(\mathbf{k}, t)$ denotes 


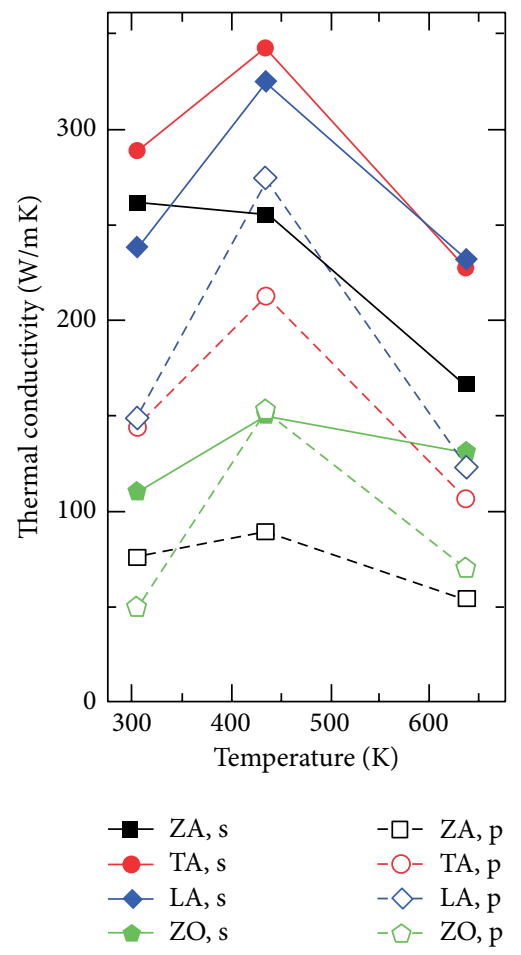

(a)

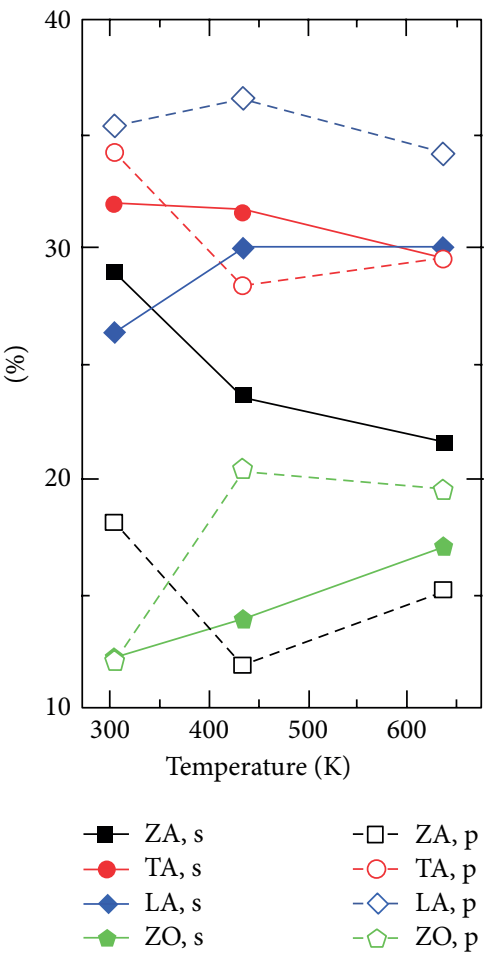

(b)

FIGURE 14: (a) Contributions (color online) and (b) the corresponding percentages of thermal conductivity from ZA, TA, LA, and TO modes in suspended ("s") and supported ("p") SLG at different temperatures. Reprinted with permission from [140].

the contribution of the $b$ th basis atom in $\alpha$ direction to the total normal mode with

$$
q_{\alpha}^{b}(\mathbf{k}, t)=\sum_{l}^{N_{c}} \sqrt{\frac{m_{b}}{N_{c}}} u_{\alpha}^{l, b}(t) \exp \left[i \mathbf{k} \cdot \mathbf{r}_{0}^{l}\right] .
$$

In (33), the time history of the atomic position displacement $u(t)$ is extracted from MD simulation, and the eigenvector $e$ is obtained from LD calculations.

4.2. Frequency Domain Normal Mode Analysis. Here, the frequency domain normal mode analysis is demonstrated by a simplified version; for detailed derivation, see [76, 77, 150]. Staring from (32), we have the spectral energy density (SED):

$$
\begin{aligned}
\Phi_{\lambda}(\omega) & =\left|\dot{q}_{\lambda}(\omega)\right|^{2}=\left|\int_{0}^{+\infty} \dot{q}_{\lambda}(t) e^{-i \omega t} d t\right|^{2} \\
& =\frac{C_{\lambda}}{\left(\omega-\omega_{\lambda}^{A}\right)^{2}+\eta_{\lambda}^{2}}
\end{aligned}
$$

where $C_{\lambda}=\left(\omega_{\lambda}^{A^{2}}+\Gamma_{\lambda}^{2}\right) q_{\lambda, 0}^{2}$ is a constant related to $\lambda$. Physically, $\Phi_{\lambda}(\omega)$ is the kinetic energy of single-phonon mode $\lambda$ in the frequency domain, in contrast to (30) which is the energy in time domain. Equation (35) is actually a Lorentzian function with peak position $\omega_{\mathbf{k}, v}^{A}$ and full width at half maximum $2 \eta_{\lambda}$. By fitting this SED function as Lorentzian form, the relaxation time $\tau_{\lambda}=1 / 2 \eta_{\lambda}$ can be obtained.
In some works, the total SED function for a given wave vector $\Phi(\mathbf{k}, \omega)=\sum_{\nu} \Phi_{\lambda}(\omega)$, which is the summation of the SEDs of phonons with the same $k$ but from different phonon branches, is evaluated instead of that of each mode. Thomas et al. [77, 150] and Feng et al. [151] pointed out that the eigenvectors are unnecessary due to the orthogonality; thus,

$$
\begin{aligned}
\Phi(\mathbf{k}, \omega) & =\sum_{\nu}^{3 n} \Phi_{\lambda}(\omega) \\
& =\frac{1}{4 \pi \tau_{0}} \sum_{\alpha, b}^{3, n} \frac{m_{b}}{N_{c}}\left|\sum_{l}^{N} \int_{0}^{\tau_{0}} \dot{u}_{\alpha}^{l, b}(t) \exp \left[i \mathbf{k} \cdot \mathbf{r}_{0}^{l}-i \omega t\right]\right|^{2} \\
& =\sum_{\alpha}^{3} \sum_{b}^{n}\left|\dot{q}_{\alpha}^{b}(\mathbf{k}, \omega)\right|^{2},
\end{aligned}
$$

where $\dot{q}_{\alpha}^{b}(\mathbf{k}, \omega)$ is time derivative and Fourier Transform of (34). From (33) to (36), the eigenvector has been abandoned, and the mathematical proof of this is presented in [151].

According to Ong et al. [152], the expression (36) is equivalent to the SED functions in [68-76]. In some of the works, the mass $m_{b}$ and unit cell number $N_{c}$ in (34) are discarded for single-mass system, since the constants do not influence the fitting results of the Lorentzian function in (35), so that only atomic velocities are needed.

4.3. Discussion and Applications. Figures 10 and 11 show two examples of time-domain NMA and frequency-domain 


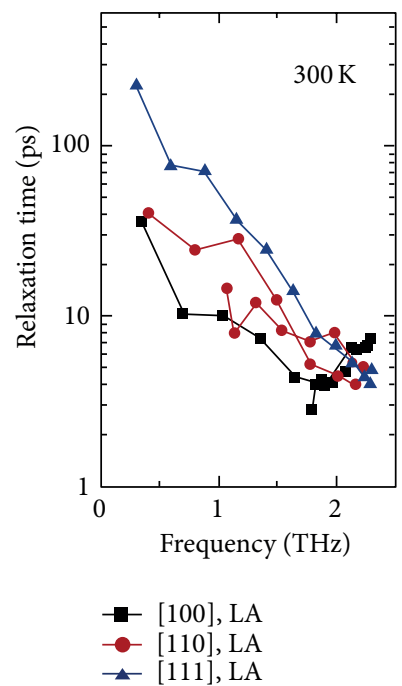

(a)

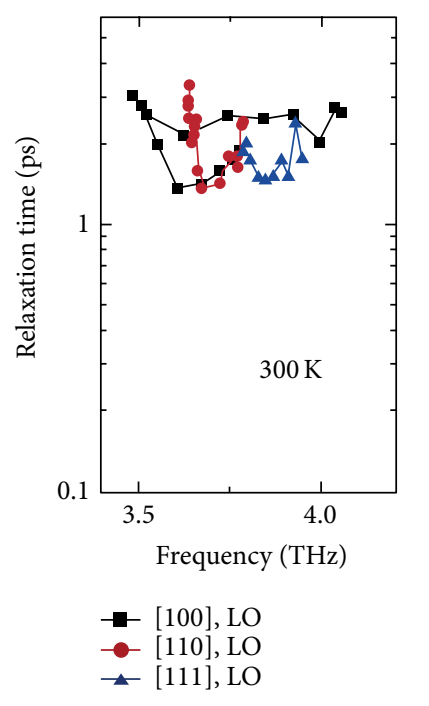

(c)
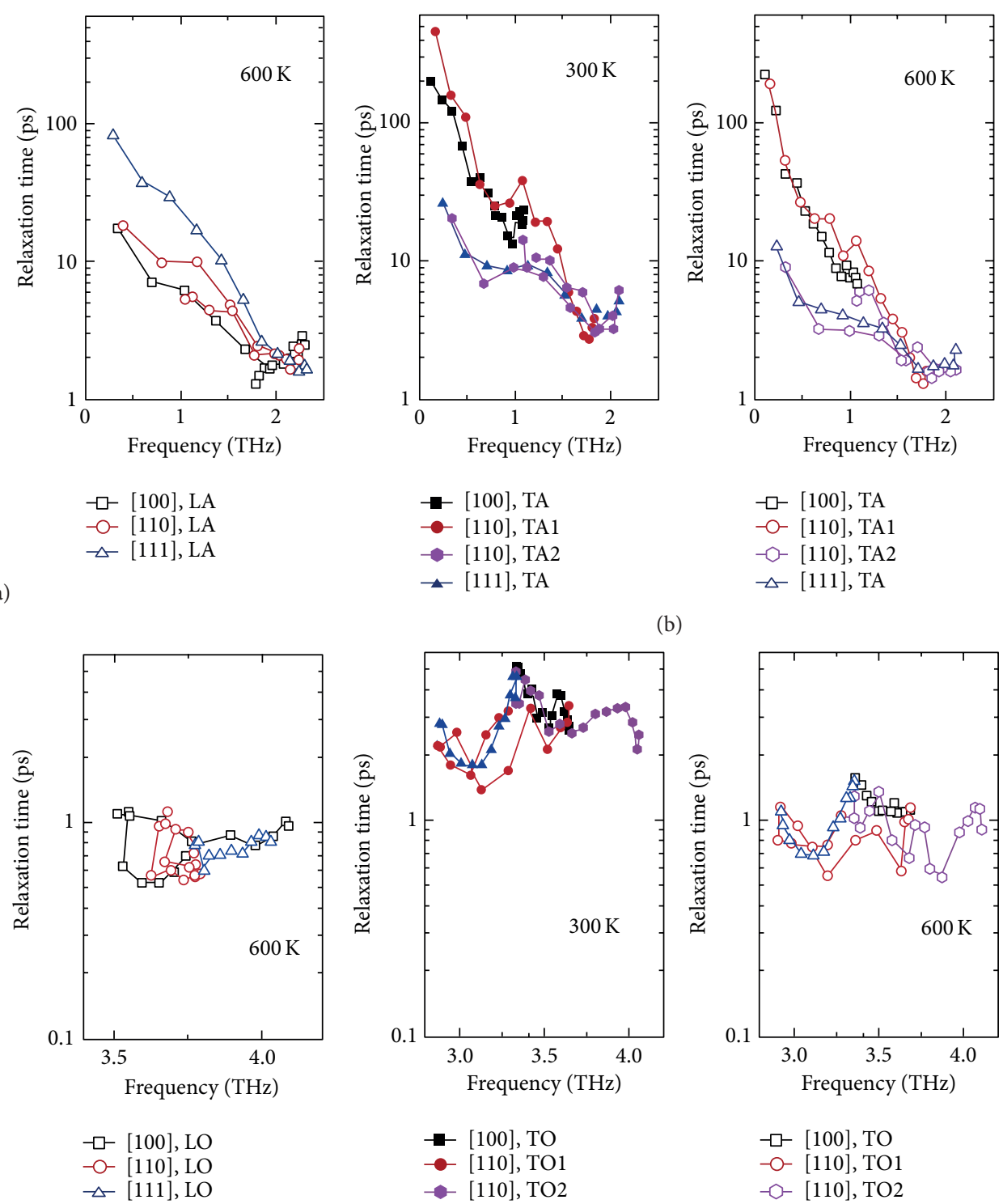

(b)
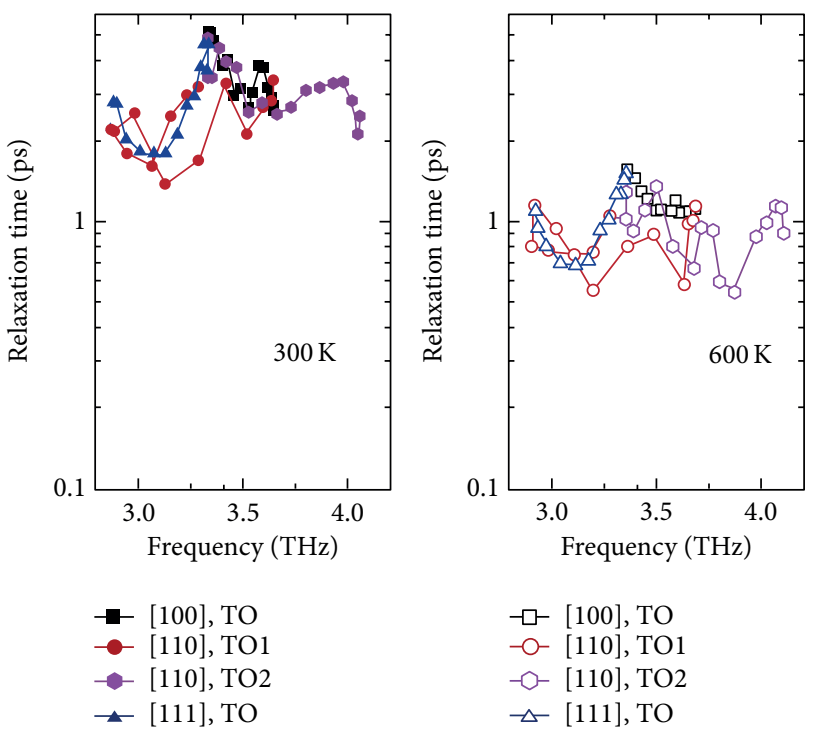

(d)

Figure 15: Spectral phonon relaxation time bulk PbTe (color online) at $300 \mathrm{~K}$ and $600 \mathrm{~K}$. Reprinted with permission from [141]. Copyright (2011), with permission from Elsevier.

NMA methods. Figure 10 presents the autocorrelation functions of total energy and potential energy of normal mode as functions of time of the TA mode of argon at $50 \mathrm{~K}$. The oscillation of the potential energy indicates that the phonon frequency and the decay rate of total energy gives the relaxation time. Figure 11 shows the SED functions of empty CNT and water-filled CNT. From the fitting of these peaks as Lorentzian functions, the phonon frequencies and relaxation times are obtained. The linewidth broadening caused by the water filled is clear from Figure 11(b).

The relaxation times predicted from MD simulation includes the effects of three-, four-, and higher-order phonon scattering processes; in contrast, ALD calculation only considers the lowest one. Thus, the ALD calculation may lose its accuracy when temperature increases, since the higherorder anharmonicity of lattice becomes greater for higher temperature due to thermal expansion. For instance, Turney et al. [102] compared the relaxation times of argon bulk predicted from the Standard SMRTA ALD calculation and the time-domain NMA at different temperatures. Figure 12 shows the inverse relaxation times of LA and TA phonon modes for argon at $20 \mathrm{~K}$ and $50 \mathrm{~K}$. We note that, at $20 \mathrm{~K}$, these two methods give reasonable agreement, whilst, at $50 \mathrm{~K}$, the ALD calculation underpredicts the scattering rate by as much as 2 or more times.

Compared to ALD calculation, MD simulation is a better tool for predicting the phonon properties of complex systems, such as the CNT filled with water and the graphene supported 


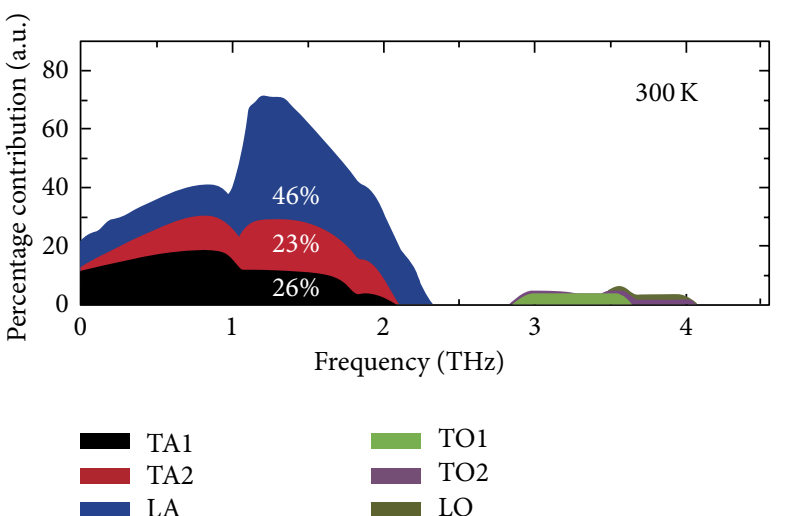

(a)

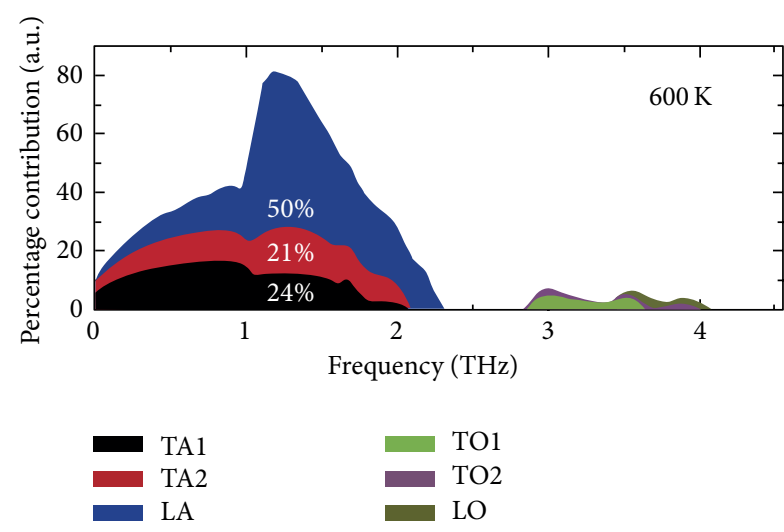

(b)

FIGURE 16: Contribution (color online) of each phonon mode to total thermal conductivity of PbTe bulk at $300 \mathrm{~K}$ and $600 \mathrm{~K}$. Reprinted with permission from [141]. Copyright (2011), with permission from Elsevier.
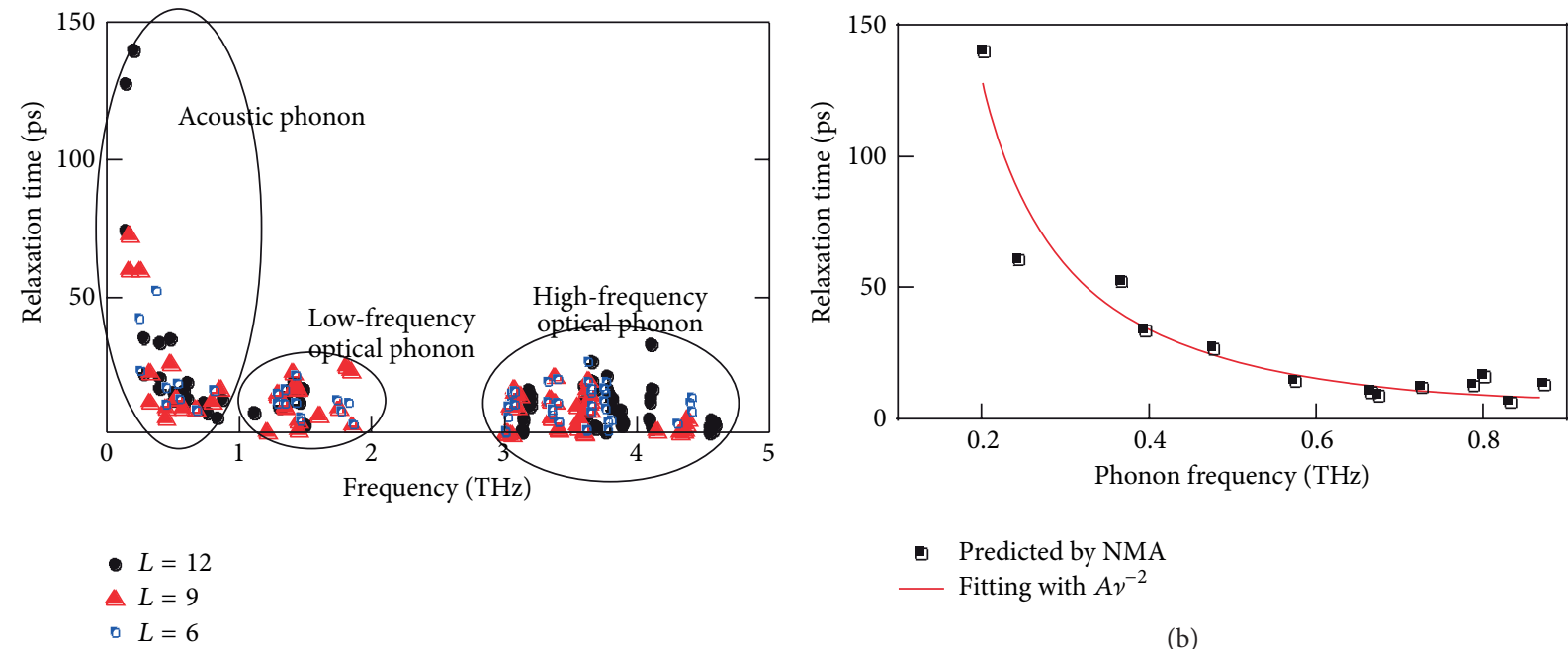

(a)

FIgURE 17: (a) Phonon relaxation times (color online) of of $\mathrm{Bi}_{2} \mathrm{Te}_{3}$ along the $\Gamma$ - $Z$ direction computed using time-domain NMA. $L$ denotes the number of cells along axis at $300 \mathrm{~K}$. (b) Phonon relaxation times of low frequency acoustic phonons along $\Gamma$ - $Z$ and the power law fitting. Reprinted with permission from [142]. Copyright 2013 by ASME.

by substrate. So far, it is hard for ALD method to handle the extrinsic phonon scattering processes other than the Umklapp scattering without fitting parameters. However, in the MD simulation, the surrounding influence is reflected by the atomic vibrating trajectory of the studied system. Qiu and Ruan $[127,128,140]$ studied the phonon transport in suspended and silicon dioxide supported SLG by frequency domain NMA with the results shown in Figures 13 and 14. We note that the flexural phonon modes ( $\mathrm{ZA}$ and $\mathrm{ZO}$ ) have much longer relaxation times than the other modes for suspended SLG, which qualitatively agree with the ALD calculation results discussed in Section 3.3.2. The MD result indicates that ZA mode contributes about $29 \%$ to the total $k$ for suspended SLG, while TA and LA modes contribute $33 \%$ and $26 \%$, respectively. Chen and Kumar [126] performed the same NMA method and obtained the similar results that ZA, TA, and LA modes contribute $23 \%, 21 \%$, and $41 \%$, respectively. The relaxation times of supported SLG are found generally shorter, by about $10 \mathrm{ps}$, than suspended SLG. This indicates that the $\mathrm{SiO}_{2}$ substrate provides strong phonon scattering by the interface and breaks down the reflection symmetry in suspended SLG. As a result, the percentage thermal conductivity contribution from ZA mode decreases about $10 \%$, while those of TA and LA modes increase about $3 \%$ and $8 \%$, respectively.

Due to the low computational complexity, the NMA methods have been applied to many cases. Time-domain NMA was used for $\operatorname{Ar}[66,67,102], S i[143,153], G e[154]$, and polyethylene $[155,156]$; in the meanwhile, frequency domain NMA has been applied to $\mathrm{Ar}$ [150], Ge [151], MgO [76], CNT [77, 78, 157], supported CNT [152], suspended and supported graphene [140], and thermoelectric materials such as $\mathrm{PbTe}$ 


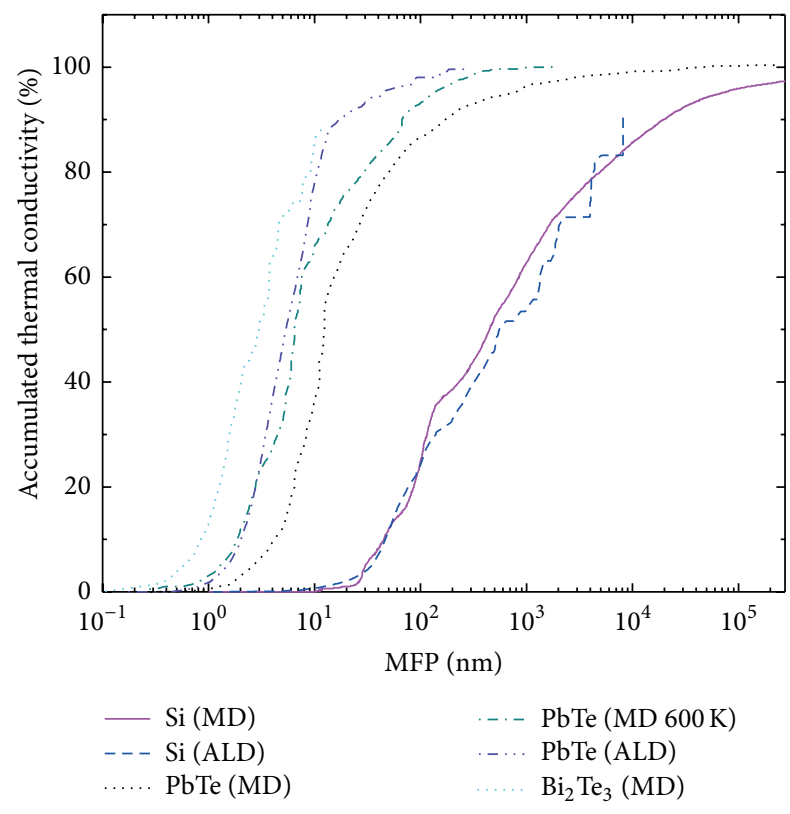

FIgURE 18: The normalized accumulated thermal conductivity (color online) of several bulk materials at room temperature as a function of phonon MFP. (Si (MD) [143]; Si (ALD) [107]; PbTe (MD) [141]; PbTe (MD $600 \mathrm{~K})$ [141]; PbTe (ALD) [106]; $\mathrm{Bi}_{2} \mathrm{Te}_{3}$ (MD) [142]).

[141] and $\mathrm{Bi}_{2} \mathrm{Te}_{3}$ [142]. So far, only few works applied NMA to defective bulk, nanowires [153], and nanoribbons. As a representative application of frequency domain NMA to bulk material, the spectral phonon relaxation times of pristine $\mathrm{PbTe}$ bulk at different temperatures in different directions are given in Figure 15. The results reveal typical features of phonon relaxation time in bulk materials: (a) acoustic phonons generally have much higher relaxation times than optical phonons, (b) for acoustic modes, the relaxation times always decrease with increasing frequency except for the high-frequency ranges which often show opposite trend, such phenomenon is also found in other materials such as argon $[67,102]$, silicon [143], and germanium [151], (c) the value of $\alpha$ in frequency dependence relation $\tau \sim \omega^{-\alpha}$ of the acoustic phonon often deviates from 2 and ranges from 0.5 to 4 , (d) $\tau$ of optical mode has weak frequency dependence, and (e) increasing temperature typically shortens the phonon relaxation time and mean free path. The order of relaxation time amplitudes of PbTe bulk at $300 \mathrm{~K}$ obtained by frequencydomain NMA agrees well with those obtained from ALD calculation in previous sections $[104,106]$. It is important to note that NMA methods do not distinguish between $N$ scattering and $U$ scattering but give a total scattering rate just as the method of ALD calculation based on Standard SMRTA. Figure 16(a) gives the contribution of each phonon mode to total thermal conductivity of PbTe bulk at $300 \mathrm{~K}$ and $600 \mathrm{~K}$. The results show that optical modes only contribute $5 \%$ to the total $k$, different with $20 \%$ given by first principle ALD calculation. The discrepancy may come from the ignorance of higher-order phonon scattering in ALD calculation. Figure 16(b) gives the accumulated $k$ 's as functions phonon MFP for Silicon at $300 \mathrm{~K}$ and PbTe at $300 \mathrm{~K}$ and $600 \mathrm{~K}$. 80\% of the total $k$ of $\mathrm{PbTe}$ is contributed by the phonons with MFP below $50 \mathrm{~nm}$, different from the value of $10 \mathrm{~nm}$ in ALD calculation [106]. This suggests that the relaxation times of low-frequency phonons predicted from ALD are longer than those from NMA, since both ALD and NMA results give reasonable total thermal conductivity. The MFPs of phonons of $\mathrm{PbTe}$ decrease roughly by a factor of 2 when temperature increases from $300 \mathrm{~K}$ to $600 \mathrm{~K}$. It is found that the phonons with MFP below $10 \mathrm{~nm}$ contribute about $32 \%$ of $k$ at $300 \mathrm{~K}$ while about $65 \%$ of $k$ at $600 \mathrm{~K}$.

The phonon properties of $\mathrm{Bi}_{2} \mathrm{Te}_{3}$ are studied by timedomain NMA [142]. The relaxation times and power law fitting of the low-frequency range are presented in Figure 17. The phonons with wavelength of $125 \mathrm{~nm}$ have relaxation time $16.9 \mathrm{~ns}$, which indicate that those phonons do not experience obvious scattering when traveling for about $400 \mathrm{ps}$ in $\mathrm{Bi}_{2} \mathrm{Te}_{3}$, consistent with experimental measurements [158]. The normalized accumulated thermal conductivity of $\mathrm{Bi}_{2} \mathrm{Te}_{3}$ as a function of phonon MFP is plotted in Figure 18. It is found that $90 \%$ and $50 \%$ of total thermal conductivity are contributed by the phonons with MFPs shorter than $10 \mathrm{~nm}$ and $3 \mathrm{~nm}$, respectively. Also shown in Figure 18 is comparison between the results from ALD calculation and MD simulation. The two curves for Si agree well with each other, while a discrepancy is found for bulk PbTe. This discrepancy may come from the inaccuracy of the interatomic potential used in performing $\mathrm{MD}$ simulation. These results are useful for the nanodesign of $\mathrm{Bi}_{2} \mathrm{Te}_{3} / \mathrm{PbTe} / \mathrm{Si}$ based thermoelectric materials in the future.

\section{Summary}

The three methods, anharmonic lattice dynamics based on Standard SMRTA, iterative anharmonic lattice dynamics, and normal mode analysis, can all predict thermal conductivity by calculating the velocities, relaxation times, and specific heats of all phonon modes. The applications are listed in Table 2, and the features of these methods are compared and listed in Table 3.

All the three methods are based on phonon Boltzmann Transport Equation and relaxation time approximation. To obtain the spectral phonon relaxation time, the first two methods calculate three-phonon scattering rates from anharmonic interatomic force constants, while the last method calculate the linewidth of spectral energy in frequency domain or the decay rate of spectral energy in time domain from molecular dynamics. Since the first two methods ignore the 4th- and higher-order phonon scattering processes, they are only valid at low temperature. The first two methods differ with each other at solving the phonon BTE: the first method assumes single mode RTA, while the second one solves the linearized BTE iteratively instead. As a result, the first method treats $N$ scattering and $U$ scattering as two independent processes that provide thermal resistance individually. However it is well known that the $N$ scattering only contribute to thermal resistance by influencing the $U$ scattering rate. The Iterative ALD remedies this error by recording all the phonon scattering processes step by step and evaluates the 
TABLE 2: Applications of the numerical methods in predicting spectral phonon properties and thermal conductivity.

\begin{tabular}{|c|c|c|c|c|}
\hline Materials & Methods ${ }^{*}$ & $\mathrm{FP}^{\dagger}$ & Reference & Year \\
\hline Ar & 1 and 3 & & {$[66]$} & 1986 \\
\hline $\mathrm{Ar}$ & 2 & & {$[46]$} & 1995 \\
\hline Ar and $\mathrm{Kr}$ & 2 & & {$[45]$} & 1996 \\
\hline Ar & 3 & & {$[67]$} & 2004 \\
\hline $\operatorname{Ar}$ & 1 and 3 & & [102] & 2009 \\
\hline Ar, Si thin films & 1 & & {$[92]$} & 2010 \\
\hline $\mathrm{C}, \mathrm{Si}$, and $\mathrm{Ge}$ & 1 & $\sqrt{ }$ & {$[43]$} & 1995 \\
\hline $\mathrm{C}$, isotope-doped $\mathrm{C}, \mathrm{Si}$, and $\mathrm{Ge}$ & 1 and 2 & $\sqrt{ }$ & {$[51]$} & 2009 \\
\hline C (Pure and natural) (extreme pressure) & 1 and 2 & & {$[62]$} & 2012 \\
\hline C nanowire & 1 and 2 & $\sqrt{ }$ & {$[64]$} & 2012 \\
\hline $\mathrm{Si}$ (isotope-doped) & 1 & & {$[136]$} & 1999 \\
\hline $\mathrm{Si}$ (isotope-doped) & 1 & & {$[91]$} & 2001 \\
\hline $\mathrm{SiC}$ & 2 & & {$[48]$} & 2002 \\
\hline $\mathrm{Si}$ and $\mathrm{Ge}$ & 1 & $\sqrt{ }$ & {$[44]$} & 2003 \\
\hline $\mathrm{Si}$ & 1 and 2 & & {$[60]$} & 2005 \\
\hline $\mathrm{Si}$ and $\mathrm{Ge}$ & 2 & $\sqrt{ }$ & {$[61]$} & 2007 \\
\hline $\mathrm{Si}$ & 3 & & [143] & 2008 \\
\hline $\mathrm{Si}$ (isotope-doped) & 2 & & {$[47]$} & 2009 \\
\hline $\mathrm{Si}$ & 1 & $\sqrt{ }$ & {$[107]$} & 2011 \\
\hline $\mathrm{Si}$ & 1 & & [99] & 2012 \\
\hline Si Nanowire & 3 & & [153] & 2009 \\
\hline $\mathrm{Si}, \mathrm{Ge}$ & 1 & $\sqrt{ }$ & {$[110]$} & 2010 \\
\hline $\mathrm{Si} / \mathrm{Ge}, M_{1} / M_{2} \mathrm{SLs}$ & 1 and 2 & & {$[59]$} & 2004 \\
\hline $\mathrm{Si} / \mathrm{Ge} \mathrm{SLs}$ & 1 & $\sqrt{ }$ & {$[108]$} & 2011 \\
\hline $\mathrm{Si} / \mathrm{Ge} \mathrm{SLs}$ & 1 & & {$[101]$} & 2013 \\
\hline $\mathrm{Si} / \mathrm{Ge} \mathrm{SLs}$ & 1 & $\sqrt{ }$ & [103] & 2013 \\
\hline SiGe alloys with embedded nanoparticles & 2 & $\sqrt{ }$ & {$[65]$} & 2011 \\
\hline SiGe alloys & 1 & $\sqrt{ }$ & {$[109]$} & 2011 \\
\hline $\mathrm{Si}, \mathrm{Ge}$, and $\mathrm{Si}_{0.5} \mathrm{Ge}_{0.5}$ & 1 & & {$[100]$} & 2012 \\
\hline $\mathrm{Si} / \mathrm{Ge}, \mathrm{GaAs} / \mathrm{AlAs}$, and $M_{1} / M_{2}$ SLs & 1 and 2 & $\sqrt{ }$ & {$[50]$} & 2008 \\
\hline $\mathrm{Ge}$ & 3 & & {$[154]$} & 2010 \\
\hline $\mathrm{Ge}$ & 4 & & {$[151]$} & 2013 \\
\hline Semiconductors (Groups IV, III-V, and II-VI) & 1 & & [117] & 2008 \\
\hline Graphene & 1 and 2 & & {$[54]$} & 2010 \\
\hline Graphene and graphite & 1 and 2 & & {$[55]$} & 2011 \\
\hline Graphene (supported and suspended) & 4 & & {$[140]$} & 2012 \\
\hline Graphene (free-standing and strained) & 1 & $\sqrt{ }$ & {$[105]$} & 2012 \\
\hline Graphene and graphite & 1 & $\sqrt{ }$ & {$[111]$} & 2013 \\
\hline CNT to graphene (diameter dependence) & 1 and 2 & & {$[53]$} & 2010 \\
\hline CNT & 4 & & [157] & 2006 \\
\hline CNT & 1 and 2 & & {$[52]$} & 2009 \\
\hline CNT (empty and water-filled) & 4 & & {$[77]$} & 2010 \\
\hline CNT (on amorphous silica) & 4 & & [152] & 2011 \\
\hline $\mathrm{BN}$ (pristine and isotope-doped) & 1 and 2 & & {$[56]$} & 2011 \\
\hline BN (multilayer and nanotubes) (pristine and isotope-doped) & 2 & & {$[58]$} & 2012 \\
\hline $\mathrm{Mg}_{2} \mathrm{Si}_{x} \mathrm{Sn}_{1-x}$ alloys (bulk and nanowire) & 2 & & {$[63]$} & 2012 \\
\hline Compound semiconductors ( $\mathrm{Si}, \mathrm{Ge}, \mathrm{GaAs}, \mathrm{Al}-\mathrm{V}, \mathrm{Ga}-\mathrm{V}, \mathrm{In}-\mathrm{V}, \mathrm{SiC}, \mathrm{AlN}$, etc.) & 1 and 2 & $\sqrt{ }$ & [112] & 2013 \\
\hline Ionic solids $\left(\mathrm{MgO}, \mathrm{UO}_{2}\right.$, and $\left.\mathrm{SrTiO}_{3}\right)$ & 2 & & {$[49]$} & 2011 \\
\hline $\mathrm{GaN}$ (GaAs, GaSb, and GaP) (pristine and isotope-doped) & 1 and 2 & $\sqrt{ }$ & [57] & 2012 \\
\hline
\end{tabular}


TABLE 2: Continued.

\begin{tabular}{|c|c|c|c|c|}
\hline Materials & Methods* & $\mathrm{FP}^{\dagger}$ & Reference & Year \\
\hline $\mathrm{PbTe}$ & 1 & $\sqrt{ }$ & {$[104]$} & 2012 \\
\hline $\mathrm{PbTe}$ & 4 & & {$[141]$} & 2011 \\
\hline $\mathrm{PbTe}, \mathrm{PbSe}$, and $\mathrm{PbTe}_{1-x} \mathrm{Se}_{x}$ & 1 & $\sqrt{ }$ & {$[106]$} & 2012 \\
\hline $\mathrm{Bi}_{2} \mathrm{Te}_{3}$ & 4 & & {$[142]$} & 2013 \\
\hline Heusler & 3 & & {$[120]$} & 2011 \\
\hline $\mathrm{MgO}$ & 4 & $\sqrt{ }$ & {$[76]$} & 2009 \\
\hline Polyethylene & 3 & & {$[155]$} & 2009 \\
\hline Polyethylene & 3 & & {$[156]$} & 2009 \\
\hline GaAs & 1 & $\sqrt{ }$ & {$[118]$} & 2013 \\
\hline GaAs/AlAs SLs & 1 & $\sqrt{ }$ & [119] & 2012 \\
\hline
\end{tabular}

${ }^{*}$ Methods: 1: Standard SMRTA scheme; 2: Iterative Scheme; 3: time-domain NMA; 4: frequency-domain NMA.

${ }^{\dagger} \mathrm{FP}$ : first principle.

TABLE 3: Comparison of different methods for predicting spectral phonon relaxation time and thermal conductivity.

\begin{tabular}{|c|c|c|c|c|c|c|}
\hline \multirow{3}{*}{$\begin{array}{l}\text { Methods } \\
\text { Equations }\end{array}$} & \multirow{3}{*}{$\begin{array}{c}\text { Analytical model } \\
\text { Table } 1\end{array}$} & \multicolumn{2}{|c|}{ ALD calculation } & \multicolumn{3}{|c|}{ MD simulation } \\
\hline & & \multirow{2}{*}{$\begin{array}{c}\text { Standard SMRTA } \\
\text { Equations (14), } \\
(15), \text { and (16) }\end{array}$} & \multirow{2}{*}{$\begin{array}{c}\text { Iterative Scheme } \\
\text { Equations (14), } \\
(15),(16),(17), \\
\text { and (18) }\end{array}$} & \multirow{2}{*}{$\begin{array}{c}\text { Time NMA } \\
\text { Equations (30), } \\
\text { (31), and (33) }\end{array}$} & \multicolumn{2}{|c|}{ Frequency NMA } \\
\hline & & & & & $\begin{array}{l}\text { Equations (33) } \\
\text { and (35) }\end{array}$ & $\begin{array}{l}\text { Equations (33) } \\
\text { and (36) }\end{array}$ \\
\hline \multirow[t]{2}{*}{ Characteristics } & \multirow{2}{*}{$\begin{array}{l}\text { Lots of } \\
\text { approximations, } \\
\text { need fitting } \\
\text { parameters }\end{array}$} & $\begin{array}{c}N \text { and } U \\
\text { processes are } \\
\text { independent } \\
\text { thermal resistant } \\
\text { sources }\end{array}$ & $\begin{array}{c}N \text { process does } \\
\text { not provide } \\
\text { thermal resistant } \\
\text { itself }\end{array}$ & \multicolumn{2}{|c|}{ Eigenvectors needed } & $\begin{array}{l}\text { Eigenvectors not } \\
\text { needed }\end{array}$ \\
\hline & & \multicolumn{2}{|c|}{ Need 2nd- and 3rd-order IFCs } & \multicolumn{3}{|c|}{ Need interatomic potential (or ab initio MD) } \\
\hline \multirow[t]{2}{*}{ Suitable for } & \multirow[t]{2}{*}{$\begin{array}{l}\text { Long wavelength, } \\
\text { Debye model }\end{array}$} & \multicolumn{2}{|c|}{$\begin{array}{l}\text { Low temperature higher-order } \\
\text { anharmonicity not large }\end{array}$} & \multicolumn{3}{|c|}{ Higher than Debye temperature } \\
\hline & & \multicolumn{5}{|c|}{ Temperature not too low, quantum effect negligible } \\
\hline Accuracy & Low & Medium & Higher & & Higher & \\
\hline $\begin{array}{l}\text { Computational } \\
\text { complexity }\end{array}$ & & High & Higher & & Low & \\
\hline \multirow{2}{*}{$\begin{array}{l}\text { Applications so } \\
\text { far } \\
\text { Further research }\end{array}$} & \multirow{2}{*}{$\begin{array}{l}\text { Some thermal } \\
\text { conductivity } \\
\text { analysis and } \\
\text { prediction }\end{array}$} & \multicolumn{2}{|c|}{$\begin{array}{l}\text { Pure and isotope-doped bulk, alloy } \\
\text { superlattice, nanostructures }\end{array}$} & \multicolumn{3}{|c|}{ Pure lattice Materials with surrounding influences } \\
\hline & & \multicolumn{2}{|c|}{$\begin{array}{l}\text { Temperature dependent IFCs, } 4 \text { th- } \\
\text { and higher-order phonon scattering }\end{array}$} & \multicolumn{3}{|c|}{$\begin{array}{l}\text { Accurate interatomic potential, large domain first } \\
\text { principle MD, defects, boundaries }\end{array}$} \\
\hline
\end{tabular}

$U$ scattering rates in the end. Compared to Green-Kubo MD (GK-MD) and Nonequilibrium MD (NEMD), these three methods give deeper insight into the thermal conductivity: the spectral phonon velocity, relaxation time, and mean free path, and the contribution of each phonon mode to thermal conductivity, which can guide the nanodesign. For accuracy and capability, the $a b$ initio ALD calculations are better than GK-MD and NEMD, since calculating $a b$ initio 3rd-order IFCs is much easier than implementing $a b$ initio MD. The limitations of the normal mode analysis are as follows: (1) it cannot distinguish $U$ and $N$ processes and (2) it is of classical nature so it cannot accurately capture the quantum distribution function (Bose-Einstein distribution) for high Debyetemperature materials at relatively low temperatures (such as graphene and CNT at room temperature). The disadvantage of these three methods is the much computational cost. Compared to analytical models, these methods do not rely on adjustable fitting parameters and thus give more reliable and accurate predictions.

These numerical methods have been applied to numerous materials and structures and revealed lots of physical nature that has never been reached before. The acoustic phonons are verified to have the $\sim \omega^{-\alpha}$ frequency dependence which agrees with earlier analytical models, while the facts that the value of $\alpha$ varies from 0 to 4 at low frequency and that the frequency dependence becomes weak and abnormal at high frequency were not observed clearly before. The optical modes are found to carry very little heat but contribute much to the scattering of acoustic phonons and thus are essential to thermal transport. In layer-/tube-structured materials, the strict selection rule of phonon scattering because reflection symmetry severely blocks the scattering of flexural acoustic phonons and thus causes extremely high relaxation time and then high thermal conductivity. In short-period superlattice, 
the large gaps between acoustic and optical phonon branches make the scattering rarely happen and thus lead to high thermal conductivity, even higher than its corresponding pure materials. These methods are also applied to defected and alloy materials using virtual crystal approach. Despite these applications, further work is still needed to predict spectral phonon properties more accurately and efficiently, such as considering the temperature-dependent IFCs and higherorder anharmonicities in ALD calculations, implementing large domain $a b$ initio molecular dynamics for normal mode analysis.

\section{Appendix}

\section{A.}

The mathematic preparations are

$$
\begin{aligned}
& \omega+\omega^{\prime}=\omega^{\prime \prime} \text { process, } \\
& \left(n_{\lambda}^{0}+1\right)\left(n_{\lambda^{\prime}}^{0}+1\right) n_{\lambda^{\prime \prime}}^{0}=n_{\lambda}^{0} n_{\lambda^{\prime}}^{0}\left(n_{\lambda^{\prime \prime}}^{0}+1\right), \\
& \omega-\omega^{\prime}=\omega^{\prime \prime} \text { process, } \\
& \left(n_{\lambda}^{0}+1\right) n_{\lambda^{\prime}}^{0} n_{\lambda^{\prime \prime}}^{0}=n_{\lambda}^{0}\left(n_{\lambda^{\prime}}^{0}+1\right)\left(n_{\lambda^{\prime \prime}}^{0}+1\right),
\end{aligned}
$$

taking advantage of $n^{0}=\left[\exp \left(\hbar \omega / k_{B} T\right)-1\right]^{-1}$.

Relaxation time approximation assumes

$$
n_{\lambda}^{\prime}=-\Psi_{\lambda} \frac{\partial n_{\lambda}^{0}}{\partial\left(\hbar \omega_{\lambda}\right)}=\Psi_{\lambda} \cdot \frac{1}{k_{B} T} n_{\lambda}^{0}\left(n_{\lambda}^{0}+1\right) .
$$

The expression of $\Psi_{\lambda}$ is obtained from the single-mode approximation (11)

$$
\Psi_{\lambda}=\tau_{\lambda} \frac{\hbar \omega_{\lambda}}{T} \mathbf{v}_{\lambda} \cdot \nabla T
$$

A.1. Standard SMRTA: The Derivation from (12) to (15). In Standard SMRTA, only $\lambda$ mode has perturbation:

$$
\begin{gathered}
n_{\lambda}=n_{\lambda}^{0}+n_{\lambda}^{\prime}, \\
n_{\lambda^{\prime}}=n_{\lambda^{\prime}}^{0}, \\
n_{\lambda^{\prime \prime}}=n_{\lambda^{\prime \prime}}^{0} .
\end{gathered}
$$

Substituting (A.5) and (A.3) into (12) with the help of (A.1), we get

$$
\begin{gathered}
n_{\lambda} n_{\lambda^{\prime}}\left(1+n_{\lambda^{\prime \prime}}\right)-\left(1+n_{\lambda}\right)\left(1+n_{\lambda^{\prime}}\right) n_{\lambda^{\prime \prime}} \\
=n_{\lambda^{\prime}}\left[n_{\lambda^{\prime}}^{0}\left(1+n_{\lambda^{\prime \prime}}^{0}\right)-\left(1+n_{\lambda^{\prime}}^{0}\right) n_{\lambda^{\prime \prime}}^{0}\right] \\
=n_{\lambda^{\prime}}\left(n_{\lambda^{\prime}}^{0}-n_{\lambda^{\prime \prime}}^{0}\right) \\
=\Psi_{\lambda} \cdot \frac{n_{\lambda}^{0}\left(n_{\lambda}^{0}+1\right)\left(n_{\lambda^{\prime}}^{0}-n_{\lambda^{\prime \prime}}^{0}\right)}{k_{B} T} .
\end{gathered}
$$

And, with the help of (A.2), we get

$$
\begin{aligned}
& n_{\lambda}\left(1+n_{\lambda^{\prime}}\right)\left(1+n_{\lambda^{\prime \prime}}\right)-\left(1+n_{\lambda}\right) n_{\lambda^{\prime}} n_{\lambda^{\prime \prime}} \\
& =n_{\lambda^{\prime}}\left[\left(1+n_{\lambda^{\prime}}^{0}\right)\left(1+n_{\lambda^{\prime \prime}}^{0}\right)-n_{\lambda^{\prime}}^{0} n_{\lambda^{\prime \prime}}^{0}\right] \\
& =n_{\lambda^{\prime}}\left(1+n_{\lambda^{\prime}}^{0}+n_{\lambda^{\prime \prime}}^{0}\right) \\
& =\Psi_{\lambda} \cdot \frac{n_{\lambda}^{0}\left(n_{\lambda}^{0}+1\right)\left(1+n_{\lambda^{\prime}}^{0}+n_{\lambda^{\prime \prime}}^{0}\right)}{k_{B} T} .
\end{aligned}
$$

From (A.6) and (A.8), we reach the relation $\partial n_{\lambda \prime} / \partial t \sim n_{\lambda \prime}$ and, compared with (11), we obtain (15). From (A.7) and (A.9), the expression of $\Psi_{\lambda}$ is obtained, the same with (A.4).

A.2. Iterative Scheme: The Derivation from (12) to (17). The Iterative Scheme solves phonon BTE (12) by assuming

$$
\begin{aligned}
& n_{\lambda}=n_{\lambda}^{0}+n_{\lambda^{\prime}}, \\
& n_{\lambda^{\prime}}=n_{\lambda^{\prime}}^{0}+n_{\lambda^{\prime}}^{\prime}, \\
& n_{\lambda^{\prime \prime}}=n_{\lambda^{\prime \prime}}^{0}+n_{\lambda^{\prime \prime}}^{\prime},
\end{aligned}
$$

where $n_{\lambda^{\prime}}^{\prime}$ and $n_{\lambda^{\prime \prime}}^{\prime}$ have the same form as $n_{\lambda}^{\prime}$ :

$$
\begin{gathered}
n_{\lambda^{\prime}}^{\prime}=-\Psi_{\lambda^{\prime}} \frac{\partial n_{\lambda^{\prime}}^{0}}{\partial\left(\hbar \omega_{\lambda^{\prime}}\right)}=\Psi_{\lambda^{\prime}} \cdot \frac{1}{k_{B} T} n_{\lambda^{\prime}}^{0}\left(n_{\lambda^{\prime}}^{0}+1\right), \\
n_{\lambda^{\prime \prime}}^{\prime}=-\Psi_{\lambda^{\prime \prime}} \frac{\partial n_{\lambda^{\prime \prime}}^{0}}{\partial\left(\hbar \omega_{\lambda^{\prime \prime}}\right)}=\Psi_{\lambda^{\prime \prime}} \cdot \frac{1}{k_{B} T} n_{\lambda^{\prime \prime}}^{0}\left(n_{\lambda^{\prime \prime}}^{0}+1\right), \\
\Psi_{\lambda^{\prime}}=\tau_{\lambda^{\prime}} \frac{\hbar \omega_{\lambda^{\prime}}}{T} \mathbf{v}_{\lambda^{\prime}} \cdot \nabla T \\
\Psi_{\lambda^{\prime \prime}}=\tau_{\lambda^{\prime \prime}} \frac{\hbar \omega_{\lambda^{\prime \prime}}}{T} \mathbf{v}_{\lambda^{\prime \prime}} \cdot \nabla T .
\end{gathered}
$$

Substituting (A.3), (A.10), (A.11), and (A.12) into (12) with the help of (A.1), abandoning the higher order terms $\Psi_{\lambda} \Psi_{\lambda^{\prime}}$, $\Psi_{\lambda} \Psi_{\lambda^{\prime \prime}}$, and $\Psi_{\lambda^{\prime}} \Psi_{\lambda^{\prime \prime}}$, we have

$$
\begin{array}{r}
n_{\lambda} n_{\lambda^{\prime}}\left(1+n_{\lambda^{\prime \prime}}\right)-\left(1+n_{\lambda}\right)\left(1+n_{\lambda^{\prime}}\right) n_{\lambda^{\prime \prime}} \\
=\frac{\left(\Psi_{\lambda}+\Psi_{\lambda^{\prime}}-\Psi_{\lambda^{\prime \prime}}\right) n_{\lambda}^{0} n_{\lambda^{\prime}}^{0}\left(1+n_{\lambda^{\prime \prime}}^{0}\right)}{k_{B} T}
\end{array}
$$

and, with the help of (A.2), we have

$$
\begin{aligned}
& n_{\lambda}\left(1+n_{\lambda^{\prime}}\right)\left(1+n_{\lambda^{\prime \prime}}\right)-\left(1+n_{\lambda}\right) n_{\lambda^{\prime}} n_{\lambda^{\prime \prime}} \\
& =\frac{\left(\Psi_{\lambda}-\Psi_{\lambda^{\prime}}-\Psi_{\lambda^{\prime \prime}}\right) n_{\lambda}^{0}\left(1+n_{\lambda^{\prime}}^{0}\right)\left(1+n_{\lambda^{\prime \prime}}^{0}\right)}{k_{B} T} .
\end{aligned}
$$

Substituting (A.4), (A.13), and (A.14) into (A.15) and (A.16), we obtain the results (17) and (18).

\section{Conflict of Interests}

The authors declare that there is no conflict of interests regarding the publication of this paper. 


\section{Acknowledgments}

The authors would like to thank the National Science Foundation, Air Force Office of Scientific Research, and the Purdue Network for Computational Nanotechnology (NCN) for the partial support.

\section{References}

[1] R. Venkatasubramanian, E. Siivola, T. Colpitts, and B. O’Quinn, "Thin-film thermoelectric devices with high room-temperature figures of merit," Nature, vol. 413, no. 6856, pp. 597-602, 2001.

[2] A. Balandin and K. L. Wang, "Significant decrease of the lattice thermal conductivity due to phonon confinement in a freestanding semiconductor quantum well," Physical Review B: Condensed Matter and Materials Physics, vol. 58, no. 3, pp. 15441549, 1998.

[3] A. Khitun, A. Balandin, and K. L. Wang, "Modification of the lattice thermal conductivity in silicon quantum wires due to spatial confinement of acoustic phonons," Superlattices and Microstructures, vol. 26, no. 3, pp. 181-193, 1999.

[4] J. Zou and A. Balandin, "Phonon heat conduction in a semiconductor nanowire," Journal of Applied Physics, vol. 89, no. 5, pp. 2932-2938, 2001.

[5] O. L. Lazarenkova and A. A. Balandin, "Electron and phonon energy spectra in a three-dimensional regimented quantum dot superlattice," Physical Review B: Condensed Matter and Materials Physics, vol. 66, Article ID 245319, 2002.

[6] N. Yang, G. Zhang, and B. Li, "Ultralow thermal conductivity of isotope-doped silicon nanowires," Nano Letters, vol. 8, no. 1, pp. 276-280, 2008.

[7] M. Hu and D. Poulikakos, "Si/Ge superlattice nanowires with ultralow thermal conductivity," Nano Letters, vol. 12, no. 11, pp. 5487-5494, 2012.

[8] W. S. Capinski, H. J. Maris, T. Ruf, M. Cardona, K. Ploog, and D. S. Katzer, "Thermal-conductivity measurements of GaAs/AlAs superlattices using a picosecond optical pump-andprobe technique," Physical Review B, vol. 59, no. 12, pp. 8106$8113,1999$.

[9] H. Fang, T. Feng, H. Yang, X. Ruan, and Y. Wu, "Synthesis and thermoelectric properties of compositional-modulated lead telluride-bismuth telluride nanowire heterostructures," Nano Letters, vol. 13, no. 5, pp. 2058-2063, 2013.

[10] K. S. Novoselov, A. K. Geim, S. V. Morozov et al., "Electric field in atomically thin carbon films," Science, vol. 306, no. 5696, pp. 666-669, 2004.

[11] K. S. Novoselov, A. K. Geim, S. V. Morozov et al., "Twodimensional gas of massless Dirac fermions in graphene," Nature, vol. 438, no. 7065, pp. 197-200, 2005.

[12] Y. Zhang, J. W. Tan, H. L. Störmer, and P. Kim, "Experimental observation of the quantum Hall effect and Berry's phase in graphene," Nature, vol. 438, no. 7065, pp. 201-204, 2005.

[13] A. K. Geim and K. S. Novoselov, "The rise of graphene," Nature Materials, vol. 6, no. 3, pp. 183-191, 2007.

[14] C. Yu, L. Shi, Z. Yao, D. Li, and A. Majumdar, "Thermal conductance and thermopower of an individual single-wall carbon nanotube," Nano Letters, vol. 5, no. 9, pp. 1842-1846, 2005.

[15] E. Pop, D. Mann, Q. Wang, K. Goodson, and H. Dai, “Thermal conductance of an individual single-wall carbon nanotube above room temperature," Nano Letters, vol. 6, no. 1, pp. 96-100, 2006.
[16] N. Hamada, S.-I. Sawada, and A. Oshiyama, "New onedimensional conductors: graphitic microtubules," Physical Review Letters, vol. 68, no. 10, pp. 1579-1581, 1992.

[17] S. Reich, C. Thomsen, and J. Maultzsch, Carbon Nan-Otubes: Basic Concepts and Physical Properties, Wiley-VCH, Weinheim, Germany, 2004.

[18] J. Hu, X. Ruan, and Y. P. Chen, “Thermal conductivity and thermal rectification in graphene nanoribbons: a molecular dynamics study," Nano Letters, vol. 9, no. 7, pp. 2730-2735, 2009.

[19] J. H. Seol, I. Jo, A. L. Moore et al., "Two-dimensional phonon transport in supported graphene," Science, vol. 328, no. 5975, pp. 213-216, 2010.

[20] A. A. Balandin, S. Ghosh, W. Bao et al., "Superior thermal conductivity of single-layer graphene," Nano Letters, vol. 8, no. 3, pp. 902-907, 2008.

[21] A. A. Balandin, "Thermal properties of graphene and nanostructured carbon materials," Nature Materials, vol. 10, no. 8, pp. 569-581, 2011.

[22] D. Nika and A. A. Balandin, "Two-dimensional phonon transport in graphene," Journal of Physics: Condensed Matter, vol. 24, no. 23, Article ID 233203, 2012.

[23] A. A. Balandin and D. Nika, "Phononics in low-dimensional materials," Materials Today, vol. 15, no. 6, pp. 266-275, 2012.

[24] N. Mingo, "Calculation of Si nanowire thermal conductivity using complete phonon dispersion relations," Physical Review B: Condensed Matter and Materials Physics, vol. 68, no. 11, Article ID 113308, 2003.

[25] N. Mingo, L. Yang, D. Li, and A. Majumdar, "Predicting the Thermal Conductivity of Si and Ge Nanowires," Nano Letters, vol. 3, no. 12, pp. 1713-1716, 2003.

[26] P. Martin, Z. Aksamija, E. Pop, and U. Ravaioli, "Impact of phonon-surface roughness scattering on thermal conductivity of thin Si nanowires," Physical Review Letters, vol. 102, no. 12, Article ID 125503, 2009.

[27] Y. Chen, D. Li, J. R. Lukes, and A. Majumdar, "Monte Carlo simulation of silicon nanowire thermal conductivity," Journal of Heat Transfer, vol. 127, no. 10, pp. 1129-1137, 2005.

[28] I. Ponomareva, D. Srivastava, and M. Menon, "Thermal conductivity in thin silicon nanowires: phonon confinement effect," Nano Letters, vol. 7, no. 5, pp. 1155-1159, 2007.

[29] T. Markussen, A.-P. Jauho, and M. Brandbyge, "Heat conductance is strongly anisotropic for pristine silicon nanowires," Nano Letters, vol. 8, no. 11, pp. 3771-3775, 2008.

[30] P. N. Martin, Z. Aksamija, E. Pop, and U. Ravaioli, "Reduced thermal conductivity in nanoengineered rough $\mathrm{Ge}$ and GaAs nanowires," Nano Letters, vol. 10, no. 4, pp. 1120-1124, 2010.

[31] C. W. Padgett, O. Shenderova, and D. W. Brenner, "Thermal conductivity of diamond nanorods: molecular simulation and scaling relations," Nano Letters, vol. 6, no. 8, pp. 1827-1831, 2006.

[32] J. F. Moreland, J. B. Freund, and G. Chen, “The disparate thermal conductivity of carbon nanotubes and diamond nanowires studied by atomistic simulation," Microscale Thermophysical Engineering, vol. 8, no. 1, pp. 61-69, 2004.

[33] P. G. Klemens, "The thermal conductivity of dielectric solids at low temperatures (theoretical)," Proceedings of the Royal Society A, vol. 208, no. 1092, pp. 108-133, 1951.

[34] P. G. Klemens, "Thermal conductivity and lattice vibrational modes," in Solid State Physics, F. Seitz and D. Turnbull, Eds., vol. 7, pp. 1-98, Academic Press, New York, NY, USA, 1958.

[35] P. G. Klemens, "The scattering of low-frequency lattice waves by static imperfections," Proceedings of the Physical Society A, vol. 68, no. 12, article 303, pp. 1113-1128, 1955. 
[36] C. Herring, "Role of low-energy phonons in thermal conduction," Physical Review, vol. 95, no. 4, pp. 954-965, 1954.

[37] M. G. Holland, "Analysis of lattice thermal conductivity", Physical Review, vol. 132, no. 6, pp. 2461-2471, 1963.

[38] H. B. G. Casimir, "Note on the conduction of heat in crystals," Physics, vol. 5, p. 495, 1938.

[39] R. Berman, F. E. Simon, and J. M. Ziman, "The thermal conductivity of diamond at low temperatures," Proceedings of the Royal Society A, vol. 220, no. 1141, pp. 171-183, 1953.

[40] R. Berman, E. L. Foster, and J. M. Ziman, "Thermal conduction in artificial sapphire crystals at low temperatures. 1: nearly perfect crystals," Proceedings of the Royal Society A, vol. 231, no. 1184, pp. 130-144, 1955.

[41] A. A. Maradudin and A. E. Fein, "Scattering of neutrons by an anharmonic crystal," Physical Review, vol. 128, no. 6, pp. 2589$2608,1962$.

[42] A. A. Maradudin, A. E. Fein, and G. H. Vineyard, "On the evaluation of phonon widths and shifts," Physica Status Solidi B, vol. 2, no. 11, pp. 1479-1492, 1962.

[43] A. Debernardi, S. Baroni, and E. Molinari, "Anharmonic phonon lifetimes in semiconductors from density-functional perturbation theory," Physical Review Letters, vol. 75, no. 9, pp. 1819-1822, 1995.

[44] G. Deinzer, G. Birner, and D. Strauch, "Ab initio calculation of the linewidth of various phonon modes in germanium and silicon," Physical Review B: Condensed Matter and Materials Physics, vol. 67, no. 14, Article ID 144304, pp. 1443041-1443046, 2003.

[45] M. Omini and A. Sparavigna, "Beyond the isotropic-model approximation in the theory of thermal conductivity," Physical Review B: Condensed Matter and Materials Physics, vol. 53, no. 14, pp. 9064-9073, 1996.

[46] M. Omini and A. Sparavigna, "An iterative approach to the phonon Boltzmann equation in the theory of thermal conductivity," Physica B: Physics of Condensed Matter, vol. 212, no. 2, pp. 101-112, 1995.

[47] J. A. Pascual-Gutiérrez, J. Y. Murthy, and R. Viskanta, “Thermal conductivity and phonon transport properties of silicon using perturbation theory and the environment-dependent interatomic potential," Journal of Applied Physics, vol. 106, no. 6, Article ID 063532, 2009.

[48] A. Sparavigna, "Lattice thermal conductivity in cubic silicon carbide," Physical Review B: Condensed Matter and Materials Physics, vol. 66, no. 17, Article ID 174301, 2002.

[49] A. Chernatynskiy, J. E. Turney, A. J. H. McGaughey, C. H. Amon, and S. R. Phillpot, "Phonon-mediated thermal conductivity in ionic solids by lattice dynamics-based methods," Journal of the American Ceramic Society, vol. 94, no. 10, pp. 3523-3531, 2011.

[50] A. Ward and D. A. Broido, "Intrinsic lattice thermal conductivity of Si/Ge and GaAs/AlAs superlattices," Physical Review B: Condensed Matter and Materials Physics, vol. 77, no. 24, Article ID 245328, 2008.

[51] A. Ward, D. A. Broido, D. A. Stewart, and G. Deinzer, "Ab initio theory of the lattice thermal conductivity in diamond," Physical Review B: Condensed Matter and Materials Physics, vol. 80, no. 12, Article ID 125203, 2009.

[52] L. Lindsay, D. A. Broido, and N. Mingo, "Lattice thermal conductivity of single-walled carbon nanotubes: beyond the relaxation time approximation and phonon-phonon scattering selection rules," Physical Review B: Condensed Matter and Materials Physics, vol. 80, no. 12, Article ID 125407, 2009.
[53] L. Lindsay, D. A. Broido, and N. Mingo, "Diameter dependence of carbon nanotube thermal conductivity and extension to the graphene limit," Physical Review B: Condensed Matter and Materials Physics, vol. 82, no. 16, Article ID 161402, 2010.

[54] L. Lindsay, D. A. Broido, and N. Mingo, "Flexural phonons and thermal transport in graphene," Physical Review B: Condensed Matter and Materials Physics, vol. 82, no. 11, Article ID 115427, 2010.

[55] L. Lindsay, D. A. Broido, and N. Mingo, "Flexural phonons and thermal transport in multilayer graphene and graphite," Physical Review B: Condensed Matter and Materials Physics, vol. 83, no. 23, Article ID 235428, 2011.

[56] L. Lindsay and D. A. Broido, "Enhanced thermal conductivity and isotope effect in single-layer hexagonal boron nitride," Physical Review B: Condensed Matter and Materials Physics, vol. 84, no. 15, Article ID 155421, 2011.

[57] L. Lindsay, D. A. Broido, and T. L. Reinecke, "Thermal conductivity and large isotope effect in GaN from first principles," Physical Review Letters, vol. 109, no. 9, Article ID 095901, 2012.

[58] L. Lindsay and D. A. Broido, "Theory of thermal transport in multilayer hexagonal boron nitride and nanotubes," Physical Review B: Condensed Matter and Materials Physics, vol. 85, no. 3, Article ID 035436, 2012.

[59] D. A. Broido and T. L. Reinecke, "Lattice thermal conductivity of superlattice structures," Physical Review B: Condensed Matter and Materials Physics, vol. 70, no. 8, Article ID 081310, 4 pages, 2004.

[60] D. A. Broido, A. Ward, and N. Mingo, "Lattice thermal conductivity of silicon from empirical interatomic potentials," Physical Review B: Condensed Matter and Materials Physics, vol. 72, no. 1, Article ID 014308, 2005.

[61] D. A. Broido, M. Malorny, G. Birner, N. Mingo, and D. A. Stewart, "Intrinsic lattice thermal conductivity of semiconductors from first principles," Applied Physics Letters, vol. 91, no. 23, Article ID 231922, 2007.

[62] D. A. Broido, L. Lindsay, and A. Ward, "Thermal conductivity of diamond under extreme pressure: a first-principles study," Physical Review B: Condensed Matter and Materials Physics, vol. 86, no. 11, Article ID 115203, 2012.

[63] W. Li, L. Lindsay, D. A. Broido, D. A. Stewart, and N. Mingo, "Thermal conductivity of bulk and nanowire $\mathrm{Mg}_{2} \mathrm{Si}_{x} \mathrm{Sn}_{1-x}$ alloys from first principles," Physical Review B: Condensed Matter and Materials Physics, vol. 86, no. 17, Article ID 174307, 2012.

[64] W. Li, N. Mingo, L. Lindsay, D. A. Broido, D. A. Stewart, and N. A. Katcho, "Thermal conductivity of diamond nanowires from first principles," Physical Review B: Condensed Matter and Materials Physics, vol. 85, no. 19, Article ID 195436, 2012.

[65] A. Kundu, N. Mingo, D. A. Broido, and D. A. Stewart, "Role of light and heavy embedded nanoparticles on the thermal conductivity of SiGe alloys," Physical Review B: Condensed Matter and Materials Physics, vol. 84, no. 12, Article ID 125426, 2011.

[66] A. J. C. Ladd, B. Moran, and W. G. Hoover, "Lattice thermal conductivity: a comparison of molecular dynamics and anharmonic lattice dynamics," Physical Review B: Condensed Matter and Materials Physics, vol. 34, no. 8, pp. 5058-5064, 1986.

[67] A. McGaughey and M. Kaviany, "Quantitative validation of the Boltzmann transport equation phonon thermal conductivity model under the single-mode relaxation time approximation," Physical Review B: Condensed Matter and Materials Physics, vol. 69, no. 9, Article ID 094303, 2004. 
[68] C. Z. Wang, C. T. Chan, and K. M. Ho, "Empirical tight-binding force modelfor molecular-dynamics simulation of Si," Physical Review B: Condensed Matter and Materials Physics, vol. 39, no. 12, pp. 8586-8592, 1989.

[69] C. Z. Wang, C. T. Chan, and K. M. Ho, "Molecular-dynamics study of anharmonic effects in silicon," Physical Review B: Condensed Matter and Materials Physics, vol. 40, no. 5, pp. 33903393, 1989.

[70] C. Z. Wang, C. T. Chan, and K. M. Ho, "Tight-binding molecular-dynamics study of phonon anharmonic effects in silicon and diamond," Physical Review B: Condensed Matter and Materials Physics, vol. 42, no. 17, pp. 11276-11283, 1990.

[71] C. Z. Wang, C. T. Chan, and K. M. Ho, "Structure and dynamics of C60 and C70 from tight-binding molecular dynamics," Physical Review B: Condensed Matter and Materials Physics, vol. 46, no. 15, pp. 9761-9767, 1992.

[72] C. Z. Wang, K. M. Ho, and C. T. Chan, "Structure and dynamics of liquid carbon," Physical Review B: Condensed Matter and Materials Physics, vol. 47, no. 22, pp. 14835-14841, 1993.

[73] C. Z. Wang, C. T. Chan, and K. M. Ho, "Tight-binding molecular-dynamics study of defects in silicon," Physical Review Letters, vol. 66, no. 2, pp. 189-192, 1991.

[74] C. Z. Wang, K. M. Ho, and C. T. Chan, "Tight-binding molecular-dynamics study of amorphous carbon," Physical Review Letters, vol. 70, no. 5, pp. 611-614, 1993.

[75] C. Z. Wang and K. M. Ho, "Structure, dynamics, and electronic properties of diamondlike amorphous carbon," Physical Review Letters, vol. 71, no. 8, pp. 1184-1187, 1993.

[76] N. de Koker, "Thermal conductivity of $\mathrm{MgO}$ periclase from equilibrium first principles molecular dynamics," Physical Review Letters, vol. 103, no. 12, Article ID 125902, 2009.

[77] J. A. Thomas, J. E. Turney, R. M. Iutzi, C. H. Amon, and A. J. H. McGaughey, "Predicting phonon dispersion relations and lifetimes from the spectral energy density," Physical Review B: Condensed Matter and Materials Physics, vol. 81, no. 8, Article ID 081411, 2010.

[78] J. A. Thomas, J. E. Turney, R. M. Iutzi, A. J. H. McGaughey, and C. H. Amon, "Predicting the phonon properties of carbon nanotubes using the spectral energy density," in Proceedings of the 2010 14th International Heat Transfer Conference (IHTC '10), IHTC14-22262, pp. 305-312, August 2010.

[79] M. Kaviany, Heat Transfer Physics, Cambridge University Press, New York, NY, USA, 2008.

[80] J. Callaway, "Model for lattice thermal conductivity at low temperatures," Physical Review, vol. 113, no. 4, pp. 1046-1051, 1959.

[81] M. Asen-Palmer, K. Bartkowski, E. Gmelin et al., "Thermal conductivity of germanium crystals with different isotopic compositions," Physical Review B: Condensed Matter and Materials Physics, vol. 56, no. 15, pp. 9431-9447, 1997.

[82] S. Tamura, "Isotope scattering of dispersive phonons in Ge," Physical Review B: Condensed Matter and Materials Physics, vol. 27, no. 2, pp. 858-866, 1983.

[83] G. P. Srivastava, The Physics of Phonons, Adam Higer, Bristol, UK, 1990.

[84] A. P. Zhernov and A. V. Inyushkin, "Kinetic coefficients in isotopically disordered crystals," Physics-Uspekhi, vol. 45, no. 5, pp. 527-552, 2002.

[85] A. V. Inyushkin, "Thermal conductivity of isotopically modified silicon: current status of research1," Inorganic Materials, vol. 38, no. 5, pp. 427-433, 2002.
[86] R. Berman, Thermal Conductivity in Solids, Oxford University Press, Oxford, UK, 1976.

[87] P. Carruthers, "Theory of thermal conductivity of solids at low temperatures," Reviews of Modern Physics, vol. 33, no. 1, pp. 92$138,1961$.

[88] M. Cardona and M. L. W. Thewalt, "Isotope effects on the optical spectra of semiconductors," Reviews of Modern Physics, vol. 77, no. 4, pp. 1173-1224, 2005.

[89] N. Mingo, D. Hauser, N. P. Kobayashi, M. Plissonnier, and A. Shakouri, "Nanoparticle-in-alloy approach to efficient thermoelectrics: silicides in SiGe," Nano Letters, vol. 9, no. 2, pp. 711-715, 2009.

[90] Z. Zhou, C. Uher, A. Jewell, and T. Caillat, "Influence of pointdefect scattering on the lattice thermal conductivity of solid solution $\mathrm{Co}\left(\mathrm{Sb}_{1-x} \mathrm{As}_{x}\right)_{3}$," Physical Review B: Condensed Matter and Materials Physics, vol. 71, no. 23, Article ID 235209, 2005.

[91] A. P. Zhernov, "The solution of the kinetic equation for phonon heat conductivity by the method of momenta and the influence of isotopic disorder on phonon heat conductivity of germanium and silicon crystals at $\mathrm{T}=300 \mathrm{~K}$," Journal of Experimental and Theoretical Physics, vol. 93, no. 5, pp. 1074-1081, 2001.

[92] J. E. Turney, A. J. H. McGaughey, and C. H. Amon, "In-plane phonon transport in thin films," Journal of Applied Physics, vol. 107, no. 2, Article ID 024317, 2010.

[93] Y. Takeda and T. P. Pearsall, "Failure of Matthiessen's rule in the calculation of carrier mobility and alloy scattering effects in Ga0.47In0.53As," Electronics Letters, vol. 17, no. 16, pp. 573-574, 1981.

[94] C. J. Glassbrenner and G. A. Slack, "Thermal conductivity of silicon and germanium from $3^{\circ}$ to the melting point," Physical Review, vol. 134, no. 4, article A1058, 1964.

[95] J. M. Ziman, Electrons and Phonons, Clarendon Press, 1962.

[96] S. Baroni, P. Giannozzi, and A. Testa, "Greens-function approach to linear response in solids," Physical Review Letters, vol. 58 , no. 18 , pp. 1861-1864, 1987.

[97] X. Gonze, "Perturbation expansion of variational principles at arbitrary order," Physical Review A: Atomic, Molecular and Optical Physics, vol. 52, no. 2, pp. 1086-1095, 1995.

[98] P. Giannozzi, S. Baroni, N. Bonini et al., "QUANTUM ESPRESSO: a modular and open-source software project for quantum simulations of materials," Journal of Physics: Condensed Matter, vol. 21, no. 39, Article ID 395502, 2009.

[99] C. Ni and J. Y. Murthy, "Phonon transport modeling using Boltzmann transport equation with anisotropic relaxation times," Journal of Heat Transfer, vol. 134, no. 8, Article ID 082401, 2012.

[100] Y. He, I. Savić, D. Donadio, and G. Galli, "Lattice thermal conductivity of semiconducting bulk materials: atomistic simulations," Physical Chemistry Chemical Physics, vol. 14, no. 47, pp. 16209-16222, 2012.

[101] I. Savić, D. Donadio, F. Gygi, and G. Galli, "Dimensionality and heat transport in Si-Ge superlattices," Applied Physics Letters, vol. 102, no. 7, Article ID 073113, 2013.

[102] J. E. Turney, E. S. Landry, A. J. H. McGaughey, and C. H. Amon, "Predicting phonon properties and thermal conductivity from anharmonic lattice dynamics calculations and molecular dynamics simulations," Physical Review B: Condensed Matter and Materials Physics, vol. 79, no. 6, Article ID 064301, 2009.

[103] I. O. Thomas and G. P. Srivastava, "Thermal conductivity of graphene and graphite," Physical Review B: Condensed Matter and Materials Physics, vol. 87, no. 11, Article ID 085410, 2013. 
[104] T. Shiga, J. Shiomi, J. Ma et al., "Microscopic mechanism of low thermal conductivity in lead telluride," Physical Review B: Condensed Matter and Materials Physics, vol. 85, no. 15, Article ID 155203, 2012.

[105] N. Bonini, J. Garg, and N. Marzari, "Acoustic phonon lifetimes and thermal transport in free-standing and strained graphen," Nano Letters, vol. 12, no. 6, pp. 2673-2678, 2012.

[106] Z. Tian, J. Garg, K. Esfarjani, T. Shiga, J. Shiomi, and G. Chen, "Phonon conduction in $\mathrm{PbSe}, \mathrm{PbTe}$, and $\mathrm{PbTe}_{1-x} \mathrm{Se}_{x}$ from firstprinciples calculations," Physical Review B: Condensed Matter and Materials Physics, vol. 85, no. 18, Article ID 184303, 2012.

[107] K. Esfarjani, G. Chen, and H. T. Stokes, "Heat transport in silicon from first-principles calculations," Physical Review B: Condensed Matter and Materials Physics, vol. 84, no. 8, Article ID 085204, 2011.

[108] J. Garg, N. Bonini, and N. Marzari, "High thermal conductivity in short-period superlattices," Nano Letters, vol. 11, no. 12, pp. 5135-5141, 2011.

[109] J. Garg, N. Bonini, B. Kozinsky, and N. Marzari, "Role of disorder and anharmonicity in the thermal conductivity of silicon-germanium alloys: a first-principles study," Physical Review Letters, vol. 106, no. 4, Article ID 045901, 2011.

[110] A. Ward and D. A. Broido, "Intrinsic phonon relaxation times from first-principles studies of the thermal conductivities of $\mathrm{Si}$ and Ge," Physical Review B: Condensed Matter and Materials Physics, vol. 81, no. 8, Article ID 085205, 2010.

[111] L. Paulatto, F. Mauri, and M. Lazzeri, "Anharmonic properties from a generalized third-order ab initio approach: yheory and applications to graphite and graphene," Physical Review B: Condensed Matter and Materials Physics, vol. 87, no. 21, Article ID 214303, 2013.

[112] L. Lindsay, D. A. Broido, and T. L. Reinecke, "Ab initio thermal transport in compound semiconductors," Physical Review B: Condensed Matter and Materials Physics, vol. 87, no. 16, Article ID 165201, 2013.

[113] W. H. Press, S. A. Teukolsky, W. T. Vetterling, and B. P. Flannery, Numerical Recipes: The Art of Scientific Computing, Cambridge University Press, Cambridge, UK, 2007.

[114] J. R. Olson, R. O. Pohl, J. W. Vandersande, A. Zoltan, T. R. Anthony, and W. F. Banholzer, "Thermal conductivity of diamond between 170 and $1200 \mathrm{~K}$ and the isotope effect," Physical Review B: Condensed Matter and Materials Physics, vol. 47, no. 22, pp. 14850-14856, 1993.

[115] L. Wei, P. K. Kuo, R. L. Thomas, T. R. Anthony, and W. F. Banholzer, "Thermal conductivity of isotopically modified single crystal diamond," Physical Review Letters, vol. 70, no. 24, pp. 3764-3767, 1993.

[116] R. Berman, "Thermal conductivity of isotopically enriched diamonds," Physical Review B: Condensed Matter and Materials Physics, vol. 45, no. 10, pp. 5726-5728, 1992.

[117] L. Lindsay and D. A. Broido, "Three-phonon phase space and lattice thermal conductivity in semiconductors," Journal of Physics: Condensed Matter, vol. 20, no. 16, Article ID 165209, 2008.

[118] T. F. Luo, J. Garg, K. Esfarjani, J. Shiomi, and G. Chen, “Gallium arsenide thermal conductivity and optical phonon relaxation times from first-principles calculations," Europhysics Letters, vol. 101, no. 1, article 16001, 2013.

[119] M. N. Luckyanova, J. Garg, K. Esfarjani et al., "Coherent phonon heat conduction in superlattices," Science, vol. 338, no. 6109, pp. 936-939, 2012.
[120] J. Shiomi, K. Esfarjani, and G. Chen, "Thermal conductivity of half-Heusler compounds from first-principles calculations," Physical Review B: Condensed Matter and Materials Physics, vol. 84, no. 10, Article ID 104302, 2011.

[121] O. Delaire, J. Ma, K. Marty et al., "Giant anharmonic phonon scattering in PbTe," Nature Materials, vol. 10, no. 8, pp. 614-619, 2011.

[122] S. N. Girard, J. He, C. Li et al., "In situ nanostructure generation and evolution within a bulk thermoelectric material to reduce lattice thermal conductivity," Nano Letters, vol. 10, no. 8, pp. 2825-2831, 2010.

[123] Z. Wang and N. Mingo, "Absence of Casimir regime in twodimensional nanoribbon phonon conduction," Applied Physics Letters, vol. 99, no. 10, Article ID 101903, 2011.

[124] N. Mingo and D. A. Broido, "Length dependence of carbon nanotube thermal conductivity and the 'problem of long waves," Nano Letters, vol. 5, no. 7, pp. 1221-1225, 2005.

[125] E. Munoz, J. Lu, and B. Yakobson, "Ballistic thermal conductance of graphene ribbons," Nano Letters, vol. 10, no. 5, pp. 16521656, 2010.

[126] L. Chen and S. Kumar, "Thermal transport in graphene supported on copper," Journal of Applied Physics, vol. 112, no. 4, Article ID 043502, 2012.

[127] B. Qiu and X. Ruan, "Mechanism of thermal conductivity reduction from suspended to supported graphene: a quantitative spectral analysis of phonon scattering," in Proceedings of the ASME 2011 Mechanical Engineering Congress and Exposition, ASME Paper IMECE2011-62963, November 2011.

[128] B. Qiu and X. Ruan, "Molecular dynamics simulations of thermal conductivity and spectral phonon relaxation time in suspended and supported graphene," http://arxiv.org/abs/1111.4613

[129] A. Alofi and G. P. Srivastava, "Phonon conductivity in graphene," Journal of Applied Physics, vol. 112, Article ID 013517, 2012.

[130] Z. Aksamija and I. Knezevic, "Thermal transport in graphene nanoribbons supported on $\mathrm{SiO}_{2}$," Physical Review B, vol. 86, no. 16, Article ID 165426, 2012.

[131] A. Y. Serov, Z. Ong, and E. Pop, "Effect of grain boundaries on thermal transport in graphene," Applied Physics Letters, vol. 102, no. 3, Article ID 033104, 2013.

[132] A. I. Cocemasov, D. L. Nika, and A. A. Balandin, "Phonons in twisted bilayer graphene," Physics Review B, vol. 88, no. 3, Article ID 035428, 2013.

[133] Z. Tian, K. Esfarjani, J. Shiomi, A. S. Henry, and G. Chen, "On the importance of optical phonons to thermal conductivity in nanostructures," Applied Physics Letters, vol. 99, no. 5, Article ID 053122, 2011.

[134] R. E. Peierls, Quantum Theory of Solids, Oxford University Press, London, UK, 1955.

[135] P. A. M. Dirac, "On the theory of quantum mechanics," Proceedings of the Royal Society A, vol. 112, no. 762, pp. 661-677, 1926.

[136] W. S. Capinski, H. J. Maris, and S. Tamura, "Analysis of the effect of isotope scattering on the thermal conductivity of crystalline silicon," Physical Review B: Condensed Matter and Materials Physics, vol. 59, no. 15, pp. 10105-10110, 1999.

[137] B. Abeles, "Lattice thermal conductivity of disordered semiconductor alloys at high temperatures," Physical Review, vol. 131, no. 5, pp. 1906-1911, 1963. 
[138] N. A. Katcho, N. Mingo, and D. A. Broido, "Lattice thermal conductivity of $\left(\mathrm{Bi}_{1-x} \mathrm{Sb}_{x}\right)_{2} \mathrm{Te}_{3}$ alloys with embedded nanoparticles," Physical Review B: Condensed Matter and Materials Physics, vol. 85, no. 11, Article ID 115208, 2012.

[139] W. A. Kamitakahara and B. N. Brockhouse, "Vibrations of a mixed crystal: neutron scattering from Ni55Pd45," Physical Review B: Condensed Matter and Materials Physics, vol. 10, no. 4, pp. 1200-1212, 1974.

[140] B. Qiu and X. Ruan, "Reduction of spectral phonon relaxation times from suspended to supported graphene," Applied Physics Letters, vol. 100, no. 19, Article ID 193101, 2012.

[141] B. Qiu, H. Bao, G. Zhang, Y. Wu, and X. Ruan, "Molecular dynamics simulations of lattice thermal conductivity and spectral phonon mean free path of PbTe: bulk and nanostructures," Computational Materials Science, vol. 53, no. 1, pp. 278-285, 2011.

[142] Y. G. Wang, B. Qiu, A. McGaughey, X. L. Ruan, and X. F. Xu, "Mode-wise thermal conductivity of bismuth telluride," Journal of Heat Transfer, vol. 135, no. 9, Article ID 091102, 6 pages, 2013.

[143] A. S. Henry and G. Chen, "Spectral phonon transport properties of silicon based on molecular dynamics simulations and lattice dynamics," Journal of Computational and Theoretical Nanoscience, vol. 5, no. 2, pp. 141-152, 2008.

[144] P. N. Keating, "Effect of invariance requirements on the elastic strain energy of crystals with application to the diamond structur," Physical Review, vol. 145, no. 2, pp. 637-645, 1966.

[145] P. N. Keating, "Theory of the third-order elastic constants of diamond-like crystals," Physical Review, vol. 149, no. 2, pp. 674678, 1966.

[146] W. Weber, "Adiabatic bond charge model for the phonons in diamond, Si, Ge, and $\alpha$-Sn," Physical Review B: Condensed Matter and Materials Physics, vol. 15, no. 10, pp. 4789-4803, 1977.

[147] K. C. Rustagi and W. Weber, "Adiabatic bond charge model for the phonons in A3B5 semiconductors," Solid State Communications, vol. 18, no. 6, pp. 673-675, 1976.

[148] M. T. Dove, Introduction to Lattice Dynamics, Cambridge University Press, New York, NY, USA, 1993.

[149] D. C. Wallace, Thermodynamics of Crystals, Wiley, New York, NY, USA, 1972.

[150] J. E. Turney, J. A. Thomas, A. J. H. McGaughey, and C. H. Amon, "Predicting phonon properties from molecular dynamics simulations using the spectral energy density," in Proceedings of the ASME/JSME 2011 8th Thermal Engineering Joint Conference, AJTEC2011-44315, March 2011.

[151] T. Feng, B. Qiu, and X. Ruan, "Role ofharmonic and anharmonic phonon eigenvectors in the phonon normal mode analysis".

[152] Z. Y. Ong, E. Pop, and J. Shiomi, "Reduction of phonon lifetimes and thermal conductivity of a carbon nanotube on amorphous silica," Physical Review B: Condensed Matter and Materials Physics, vol. 84, no. 16, Article ID 165418, 2011.

[153] D. Donadio and G. Galli, "Atomistic simulations of heat transport in silicon nanowires," Physical Review Letters, vol. 102, no. 19, Article ID 195901, 2009.

[154] J. V. Goicochea and B. Michel, "Phonon relaxation times of germanium determined by molecular dynamics at $1000 \mathrm{~K}$," in Proceedings of the IEEE/CPMT 26th Semiconductor Thermal Measurement, Modeling \& Management Symposium (SEMITHERM '10), pp. 278-282, February 2010.

[155] A. Henry and G. Chen, "Anomalous heat conduction in polyethylene chains: theory and molecular dynamics simulations," Physical Review B: Condensed Matter and Materials Physics, vol. 79, no. 14, Article ID 144305, 2009.
[156] A. Henry and G. Chen, "Explicit treatment of hydrogen atoms in thermal simulations of polyethylene," Nanoscale and Microscale Thermophysical Engineering, vol. 13, no. 2, pp. 99-108, 2009.

[157] J. Shiomi and S. Maruyama, "Non-Fourier heat conduction in a single-walled carbon nanotube: classical molecular dynamics simulations," Physical Review B: Condensed Matter and Materials Physics, vol. 73, no. 20, Article ID 205420, 2006.

[158] Y. Wang, C. Liebig, X. Xu, and R. Venkatasubramanian, "Acoustic phonon scattering in $\mathrm{Bi}_{2} \mathrm{Te}_{3} / \mathrm{Sb}_{2} \mathrm{Te}_{3}$ superlattices," Applied Physics Letters, vol. 97, no. 8, Article ID 083103, 2010. 

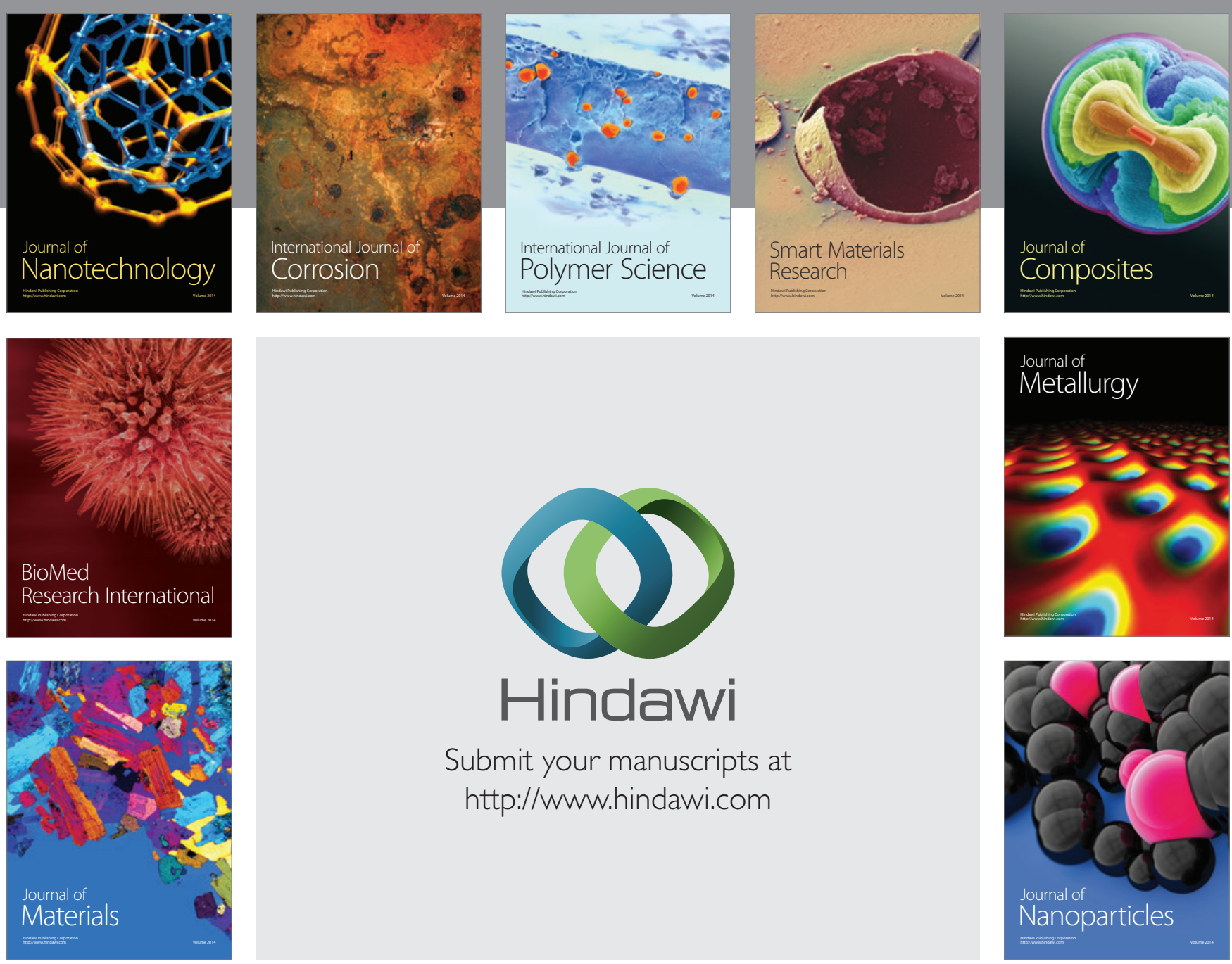

Submit your manuscripts at http://www.hindawi.com
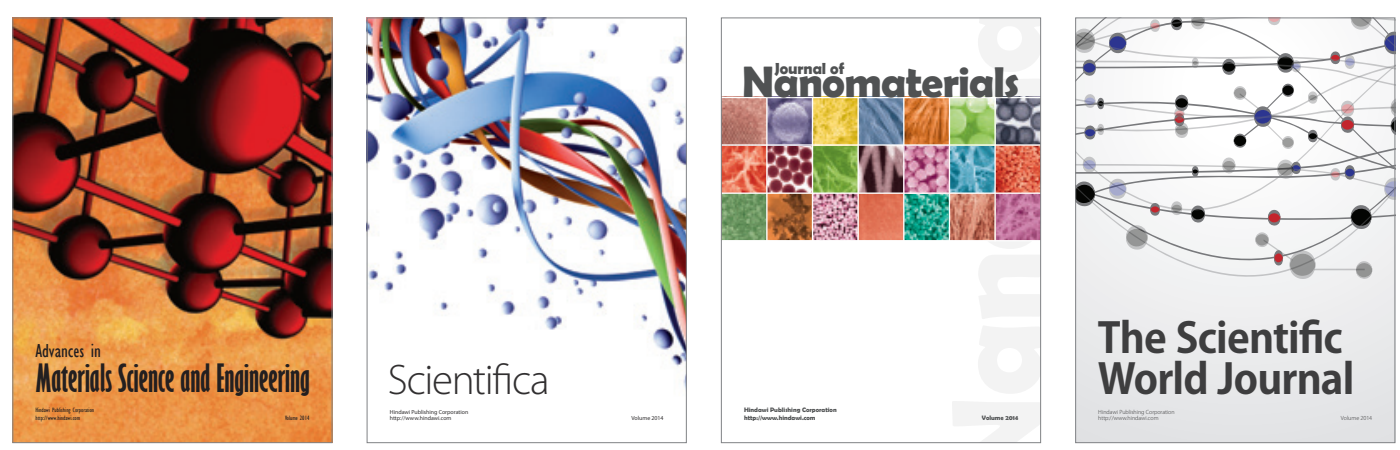

\section{The Scientific World Journal}
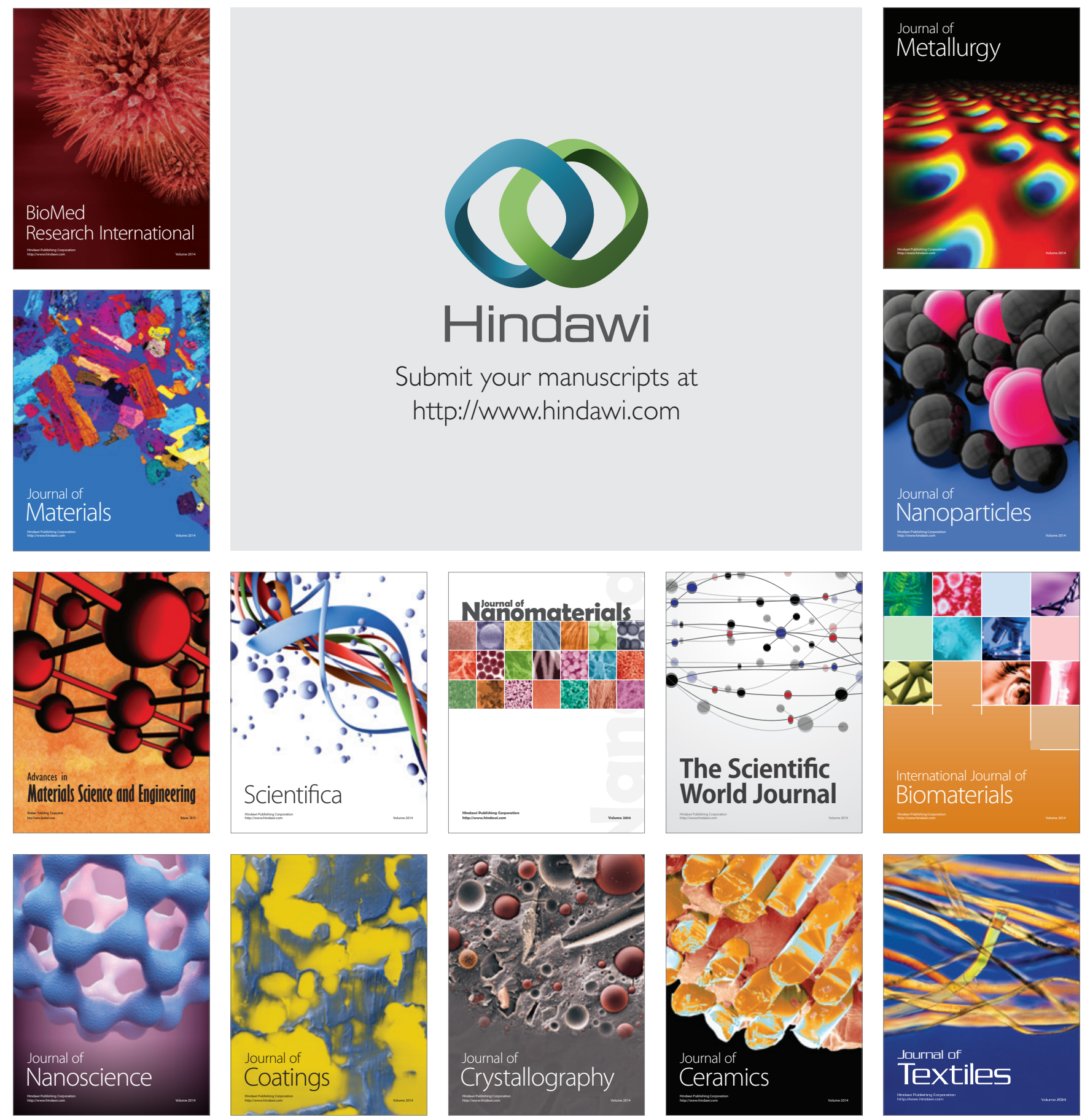